\title{
The Acquisition of Quality Information in a Supply Chain with Voluntary vs. Mandatory Disclosure
}

September 29, 2019

\begin{abstract}
Quality information acquisition and disclosure have significant ramifications for supply chain members. This paper investigates the interaction between a manufacturer's product quality information acquisition and different quality information disclosure systems in a supply chain wherein the manufacturer can privately acquire the precise quality information of his product by affordable means initially. We consider two different quality information disclosure systems for the quality information acquisition: voluntary disclosure (i.e., the manufacturer determines whether to disclose the quality information that he has acquired), and mandatory disclosure (i.e., the manufacturer is mandated to disclose the quality information that he has acquired). We examine the effects of voluntary disclosure and mandatory disclosure on the equilibrium strategies and payoffs of the manufacturer and the retailer and on the consumer surplus. It is shown that mandatory disclosure significantly reduces the manufacturer's incentive to acquire the precise product quality information and leads to a reduction in the product quality information that the retailer and the consumers can receive. Interestingly, although the manufacturer is ex-ante better off, the retailer's ex-ante payoff and the expected consumer surplus become lower under mandatory disclosure, as opposed to voluntary disclosure of product quality information.
\end{abstract}

Keywords: quality; information acquisition; voluntary disclosure; mandatory disclosure; supply chain; game theory 


\section{Introduction}

The significance of product quality is well recognized in a wide range of supply chains (e.g., food, electronic devices and automobiles). However, due to some intricate manufacturing techniques, production invisibility (e.g., outsourcing), human errors and varying environmental factors (e.g., temperature and humidity), even the manufacturer itself may be uncertain about the precise quality level of its product. To ascertain the precise quality level, in practice, a manufacturer may make efforts to acquire the precise quality information of its product via affordable means. The manufacturer may voluntarily make investment to set up a stringent internal quality management system to test product quality itself. For example, Golden State Foods, one of the largest food suppliers in the US, has invested a lot in the Internet of Things (IoT) system to detect and monitor the food quality across the entire supply chain; ${ }^{1}$ Foxconn, the primary manufacturing partner of Apple for iOS devices such as iPhone and iPad, has added expensive X-ray systems to its assembly lines to inspect the quality of products. ${ }^{2}$ Moreover, the manufacturer may incur cost to seek help from third-party certifiers to evaluate their product quality. For instance, toy manufacturers can seek ASTM International certification ${ }^{3}$, and automobile manufacturers often take part in the NTS testing $^{4}$ or the New Car Assessment Program (NCAP) ${ }^{5}$.

Despite the costs involved, manufacturers are still keen on acquiring the precise quality information of their products, as it endows them with an information advantage over the downstream parties (e.g., retailer and consumers) and allows them to craft more targeted quality information disclosure strategies. For instance, if the acquired information indicates that quality is high, the manufacturer would try hard to advocate this information via different channels (e.g., media advertising) to attract more purchases. Conversely, if quality information acquisition yields negative quality data, the manufacturer would choose to withhold this unfavorable information. Nonetheless, it is not always the manufacturer's desire to only disclose the positive quality information. Rather, for some quality information acquisition processes, the outcomes of quality information acquisition are forced to be disclosed publicly. One typical example is the New Car Assessment Program (NCAP), the most authoritative program of its kind in the world. According to its policy, participation in the NCAP is voluntary for auto manufacturers, but the test results are publicly available on its official website. ${ }^{6}$ Thus, regardless of whether the test results are positive or neg-

\footnotetext{
${ }^{1}$ www.ibm.com/blogs/events/think-2018/think-2018-presents/improving-food-safety-internet-things /

${ }^{2}$ www.macrumors.com/2012/03/05/foxconn-reportedly-increasing-quality-control-with-x-ray-imaging/

${ }^{3}$ www.astm.org/CERTIFICATION/

${ }^{4}$ www.nts.com/about/, www.nts.com/industries/automotive/

${ }^{5}$ www.globalncap.org/

${ }^{6}$ www.euroncap.com/en, http:/ /blog.twwhiteandsons.co.uk/tw-white-news/euro-ncap-explained-2/
} 
ative, auto manufacturers that participate in the program cannot hide this information from the public. Walmart and Carrefour respectively set up blockchain systems to ensure that the quality information of some products from their suppliers are transparent and credible, and to assess the product quality in real time. Once relevant suppliers participate in these blockchain systems, the product quality information that the suppliers obtain subsequently becomes transparent across the entire supply chain. ${ }^{7}$

Based on the above discussion, it is evident that the disclosure of a manufacturer's acquired product quality information can be subject to two different quality information disclosure systems. The first is voluntary disclosure in which the upstream manufacturer can decide whether to disclose its privately acquired quality information to the unknown downstream parties (i.e., the retailer and the consumers). The second is mandatory disclosure in which the manufacturer is forced to disclose all the acquired quality information publicly. Although both voluntary and mandatory disclosure systems are quite prevalent in practice, their impacts on firms' equilibrium strategies and payoffs have been seldom discussed adequately. Therefore, in this paper, we seek to conduct a novel approach regarding the comparison between voluntary disclosure of quality information and mandatory disclosure of quality information. How do the manufacturer's sequential/joint quality information acquisition and disclosure strategies change under these two quality information disclosure systems? How do firms' payoffs (i.e., manufacturer's payoff and retailer's payoff) and consumer surplus react to the manufacturer's quality information acquisition strategies under different quality information disclosure systems?

These questions are addressed in a classic supply chain setting wherein the manufacturer sells products to end consumers via an independent retailer. The product quality is initially uncertain, but the manufacturer has a chance to acquire the precise product quality information via some affordable methods to ascertain accurate quality level. If the manufacturer acquires quality information, it then needs to decide whether to disclose the outcomes from information acquisition conditional on the information disclosure system to which it is subject: voluntary disclosure or mandatory disclosure. On the other hand, the retailer and the consumers rely on the manufacturer's disclosure behavior to update their beliefs on the product quality level and make their pricing and purchasing decisions accordingly. If quality information is truthfully disclosed by the manufacturer, the retailer and the consumers can confirm the product's exact quality level; in contrast, if qualify information is not disclosed, they make rational quality inferences from the manufacturer's non-disclosure behavior.

\footnotetext{
${ }^{7}$ https:/ / techcrunch.com/2018/09/24/walmart-is-betting-on-the-blockchain-to-improve-food-safety/, www.foodbev.com/news/carrefour-enables-milk-product-traceability-through-blockchain/
} 
We demonstrate that different quality information disclosure systems generate significant ramifications for the manufacturer's voluntary quality information acquisition strategies, as well as for the retailer's pricing and the consumers' purchasing decisions. Specifically, the manufacturer's incentive to engage in quality information acquisition is strongly reduced by mandatory disclosure compared with voluntary disclosure. This effect can be explained by considering that when the acquired quality information must be disclosed, the manufacturer no longer possesses an information advantage over the retailer and the consumers; accordingly, the benefit of information acquisition in crafting the disclosure strategy vanishes. Both consequences make the manufacturer more conservative in quality information acquisition under mandatory disclosure.

Given that mandatory disclosure would discourage the manufacturer from voluntarily acquiring the precise product quality information, one may infer that the manufacturer would be worse off under mandatory disclosure of quality information than under voluntary disclosure of quality information. However, we argue just the opposite: a manufacturer can benefit more from mandatory disclosure than from voluntary disclosure. That is to say, an inflexible quality information disclosure system can improve the manufacturer's profitability. One driver of this unintended result is the strategic quality inference of the downstream parties (i.e., the retailer and the consumers) from the manufacturer's disclosure behavior. In particular, the downstream parties infer that product quality is relatively low when they observe non-disclosure under voluntary disclosure. This is because they cannot determine whether non-disclosure is driven by the manufacturer's no acquisition of quality information or by the manufacturer's intention to withhold any quality information indicating that the quality level is low. In contrast, under mandatory disclosure, non-disclosure only indicates that the manufacturer has not acquired quality information, thus the downstream parties do not have a negative impression of product quality after observing this phenomenon. In this sense, we uncover an intriguing relationship between the quality information disclosure system and the downstream parties' quality speculation process, leading to some nontrivial implications regarding the manufacturer's profitability, in a supply chain setting.

Another interesting observation is that both the retailer and the consumers are better off under voluntary disclosure than under mandatory disclosure. This is in strict contrast to conventional wisdom regarding mandatory disclosure, which is viewed as an effective approach that regulators adopt to protect consumer rights by preventing the manufacturer from hiding any negative quality information. An explanation is that although mandatory disclosure indeed eliminates information asymmetry between the upstream manufacturer and the downstream parties, it also prevents the manufacturer from acquiring quality information actively in a context where the acquisition of quality information is costly. Combining these effects together, we show that this 
downside of mandatory disclosure, that is, preventing the acquisition of quality information, is so pronounced that it eventually results in even less transparent quality information in the supply chain than that under voluntary disclosure, leading to lower payoff received by the retailer and lower consumer surplus.

Furthermore, we discuss what happens when the manufacturer can be endowed with some private product quality information (over the retailer and the consumers) ex-ante. We show that under such a circumstance, the manufacturer still has a stronger quality information acquisition incentive but obtains a lower ex-ante payoff under voluntary disclosure than under mandatory disclosure. This observation is consistent with our basic model. While from the perspectives of the retailer and the consumers, they may prefer mandatory disclosure over voluntary disclosure depending on the chance that the manufacturer knows the product quality information ex-ante. We also consider some variations about the demand function and the distributions of product quality and quality information acquisition cost, wherein the main results in the basic model still hold with these modifications. Note that the ratio between the quality information acquisition cost and the manufacturer's payoff is examined too, in which we show that even for a relatively small information acquisition cost, it can still influence the manufacturer's equilibrium information acquisition strategy significantly.

The rest of the paper is organized as follows. Section 2 reviews the related literature. Section 3 describes the model setup in detail. Section 4 presents the main analysis and results. We analyze the manufacturer's equilibrium quality information acquisition strategies under both voluntary disclosure and mandatory disclosure, and investigate the effects of these two quality information disclosure systems on the equilibrium payoffs of the firms (i.e., the manufacturer and the retailer) in the supply chain and the expected consumer surplus. Section 5 discusses extensions, and section 6 concludes the study. Detailed proofs are shown in the appendixes.

\section{Related Literature}

This study is related to the literature on quality information disclosure. Grossman and Hart (1980), Grossman (1981) and Milgrom (1981) first studied the voluntary provision of quality information and proposed the well-known "unraveling rule". Under this rule, a firm would voluntarily disclose any private quality information as long as there is zero cost to verifiably disclose the information. However, this result of information unraveling is derived under strict assumptions. Since then, researchers have examined the factors that may lead to partial disclosure of quality information. For example, Jovanovic (1982) shows that with a positive cost of quality information 
disclosure, a firm makes voluntary disclosures only if quality is sufficiently high. Sun (2011) indicates that a firm with a high-quality product is less likely to disclose product information when the product is characterized by multiple attributes. Moreover, the market structure is another factor that affects a firm's product quality information disclosure strategy (e.g., Cheong and Kim 2004, Guo and Zhao 2009, Levin et al. 2009, Ghosh and Galbreth 2013, Li and Peeters 2017). For instance, Levin et al. (2009) investigate the disclosure of quality information under duopoly and cartel structures and show that expected disclosure is higher under a cartel than under a duopoly. Guo and Zhao (2009) show that the amount of quality information that the firms disclose depends on whether firms disclose the information simultaneously or sequentially. The mandatory disclosure of quality information has also received much attention in the literature. Fishman and Hagerty (2003) show that with uninformed consumers who do not understand the disclosed quality information, mandatory information disclosure benefits the buyers (with informed customers strictly better off and uninformed customers unaffected). Board (2009) demonstrates that with fierce price competition, mandatory disclosure of product quality information can promote competition and raise consumer surplus at the expense of firm profits. In addition, the empirical literature concentrates on the impacts of the mandatory disclosure of quality information (e.g., Jin and Leslie 2003, Dafny and Dranove 2008, Forbes et al. 2015). Our study differs primarily in that the manufacturer is not exogenously informed of product quality, and the disclosed quality information must be first voluntarily acquired at a cost by the manufacturer. Thus, consideration of the strategic interaction between voluntary quality information acquisition and quality information disclosure systems is a novel approach.

This paper also contributes to the literature on the acquisition and disclosure (sharing) of information. The strategic sharing of market demand information in supply chain has been widely studied (e.g., Zhang 2002, Mishra et al. 2009, Ha et al. 2011, Shang et al. 2015, Jiang et al. 2016), where the process of demand information acquisition is exogenous and costless. Guo (2009) investigates the downstream retailer's incentive to acquire demand information and to disclose it to the upstream manufacturer. Conversely, Guo and Iyer (2010) consider a manufacturer that acquires and shares demand information, and compare the manufacturer's acquisition strategy under mandatory information sharing and voluntary information sharing. Jansen (2008) studies the market demand information acquisition and production incentives of duopolies and analyzes the consequences for firms' profits under a strategic information sharing format and pre-committed information sharing format. Li et al. (2014) investigate the retailer's disclosure strategy with respect to its information status, informed or uninformed, instead of the information content of uncertain demand, considering that information acquisition may not be successful. Gao et al. (2014) 
investigate a strategic information management issue in an export-processing trade market and find that the producer discloses the production yield rate information that it has acquired only when the yield rate is in an intermediate range. Moreover, Matthews and Postlewaite (1985) and Shavell (1994) indicate that uncertainty in information acquisition can also stop unraveling. Arya et al. (2014) study the manufacturer's production decision in a discretionary quality testing and reporting environment. They find that production cuts can improve the buyer's quality perception and dampen the manufacturer's incentive to conduct excessive quality testing. Guan and Chen (2017) study the interplay between the acquisition of consumer information and the disclosure of quality information, and find that the former has an impact on quality information disclosure by the manufacturer. Our study complements these previous studies by investigating a manufacturer's joint acquisition and disclosure strategies regarding uncertain quality information. We are interested in how different quality information disclosure systems influence the consumer's strategic quality inference process and the firms' equilibrium strategies and payoffs in a supply chain setting. We uncover several unintended results, such as the manufacturer's (retailer's) exante payoff is higher (lower) under mandatory disclosure of product quality information, which is in sharp contrast to the results that do not consider the impacts of information disclosure systems on information acquisition behavior.

\section{The Model}

Consider a supply chain with a manufacturer (he), selling a product to consumers through an independent retailer (she). Both the manufacturer and the retailer are risk-neutral and attempt to maximize their individual payoffs. The consumers have a positive willingness to pay for quality, and the market demand for the product is represented by $Q=\theta q-p$, where $q$ represents the quality of manufacturer's product, $\theta$ denotes the consumer's preference with regard to the product quality, and $p$ is the retail price charged by the retailer. ${ }^{8}$ The demand function is derived from the consumer's quadratic utility function: $U=(\theta q) Q-\frac{1}{2} Q^{2}-p Q$ (Singh and Vives, 1984), which has been widely used in the economics and management literature. The quality of manufacturer's product $q$ is a random variable, which represents that the outcome of a production process is normally influenced by some uncontrollable and random factors. Initially, the precise product quality $q$ is uncertain to the upstream manufacturer and downstream parties (i.e., the retailer and the consumers). This situation usually arises in the early stage of the product life cycle when the

\footnotetext{
${ }^{8}$ We also examine a more general form of demand function: $Q=A+\theta q-p$, where $A(>0)$ is a constant value representing the base demand, and show that all the results still hold (see section 5.2).
} 
product specifications are new. All the parties share common prior beliefs about the product quality, which follows a uniform distribution $q \sim U(0,1)$ with density function $f(\cdot)$ and distribution function $F(\cdot)$. Note that such assumptions are consistent with the related literature (e.g., Board 2009, Guo 2009, Guo and Zhao 2009, Sun 2011, Ghosh and Galbreth 2013, Guan and Chen 2015, Guan and Chen 2017, Li and Peeters 2017, Markopoulos and Hosanagar 2017). ${ }^{9}$

The manufacturer, however, can seek to acquire the precise quality information of his product at a private information acquisition cost. For example, the manufacturer may privately incur costs to set up a stringent internal product quality testing process or seek third-party certifications. The information acquisition cost may vary with the means that the manufacturer adopts to ascertain the precise level of his product quality. Thus, we use $k$ to denote the cost that the manufacturer expends on quality information acquisition and its value can be privately observed only by the manufacturer. While both the retailer and the consumers maintain their prior beliefs that the quality information acquisition cost is uniformly distributed over $[0, K]$, with density function $g(\cdot)$ and distribution function $G(\cdot) \cdot{ }^{10}$ This setting regarding the information acquisition cost is in line with the related literature (e.g., Shavell 1994, Arya et al. 2014, Li and Peeters 2017).

The manufacturer voluntarily decides whether to acquire the precise quality information of his product, whose decision is denoted by $s_{A} \in\{a, n a\}$. Once he does so $\left(s_{A}=a\right)$, he incurs quality information acquisition cost $k$ and will learn exact product quality level $q$. While if he does not acquire the quality information $\left(s_{A}=n a\right)$, he only knows the distribution $f(\cdot)$ over $q$. Then, the manufacturer may disclose the quality information that he has acquired to the downstream parties, which is dependent on the quality information disclosure system to which the quality information acquisition process is subject. First, under voluntary disclosure, the manufacturer can discretionarily determine whether to disclose the quality information that he has acquired. For example, auto manufacturers privately seek to ascertain the quality of their automobiles via NTS testing, and they can determine whether to disclose the testing results publicly. In particular, the manufacturer may disclose positive information and withhold negative information. Moreover, the manufacturer will also take the downstream parties' subsequent responses into consideration

\footnotetext{
${ }^{9}$ Note that the manufacturer may ex-ante know the quality information of his product with some probabilities. We investigate this case in section 5.1 .

${ }^{10}$ We require that the upper bound of quality information acquisition cost $K$ is higher than $\frac{\theta^{2}}{24}$ to guarantee the existence of an equilibrium when disclosure of quality information is voluntary. In practice, the process of product quality information acquisition can be complex and costly such as the quality testing of semiconductor chips (www.adapteva.com/andreas-blog/semiconductor-economics-101/) and the Internet of Things systems established by food suppliers such as Golden State Foods to acquire food quality information and assess the food quality in real time.
} 
when he makes the information disclosure decision. The retailer/consumer can observe precise product quality information if the manufacturer discloses it, which is denoted by $s_{D}=d$. Otherwise, if the manufacturer chooses non-disclosure $\left(s_{D}=n d\right)$, the retailer/consumer cannot see exact quality information, but they can make rational quality inferences from the manufacturer's non-disclosure behavior. Second, under mandatory disclosure, the precise quality information acquired by the manufacturer must be disclosed regardless whether it is positive or negative. One example is New Car Assessment Program (NCAP) for automobiles. Auto manufacturers voluntarily decide whether to take part in NCAP, while the testing results must be published publicly. Under such circumstances, the retailer/consumer can always observe exact quality information if the manufacturer engages in quality information acquisition.

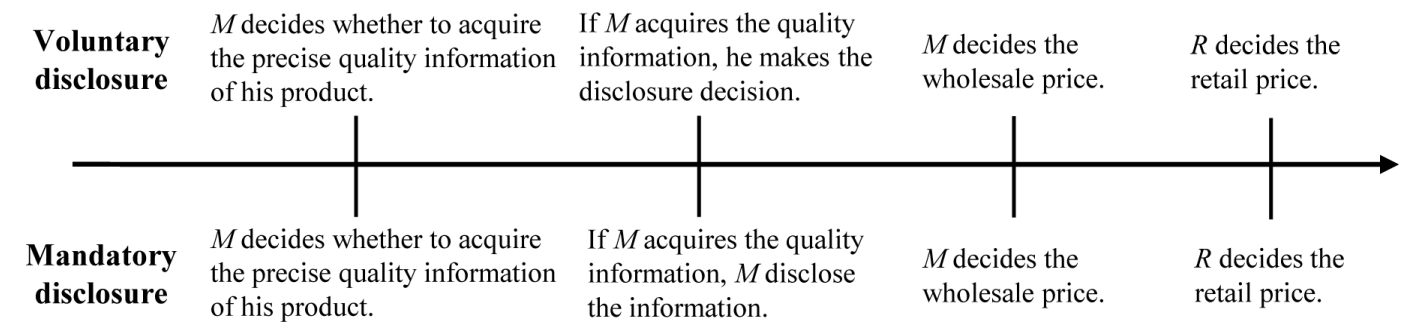

Figure 1 Sequence of events

Table 1 Summary of model notations

\begin{tabular}{cl}
\hline Notation & \multicolumn{1}{c}{ Description } \\
\hline$Q$ & Market demand \\
$\theta$ & Consumer's preference for product quality \\
$q$ & Product quality \\
$k$ & Cost of product quality information acquisition \\
$s_{A}$ & Manufacturer's quality information acquisition decision $\left(s_{A} \in\{a, n a\}\right)$ \\
$s_{D}$ & Manufacturer's quality information disclosure decision $\left(s_{D} \in\{d, n d\}\right)$ \\
$p$ & Retail price \\
$w$ & Wholesale price \\
$\Pi$ & Manufacturer's interim payoff \\
$\pi$ & Retailer's interim payoff \\
$E[\Pi]$ & Manufacturer's ex-ante payoff \\
$E[\pi]$ & Retailer's ex-ante payoff \\
$C S$ & Consumer surplus \\
$E C S$ & Expected consumer surplus \\
$\sim$ & Voluntary disclosure \\
$\sim$ & Mandatory disclosure \\
\hline
\end{tabular}


The sequence of events is illustrated in Figure 1, where $M$ represents the manufacturer and $R$ represents the retailer. First, the manufacturer privately observes the cost of quality information acquisition $k$ and decides whether to acquire the precise quality information of his product. Second, given the quality information disclosure system, the manufacturer makes the disclosure decision. In particular, under voluntary disclosure, he decides whether to disclose the quality information that he has acquired. Under mandatory disclosure, the manufacture is required to fully disclose the quality information once he acquires it. Third, the manufacturer sets the wholesale price for each unit of product. Finally, the retailer charges the retail price. The model notations are provided in Table 1.

It is worth mentioning that in line with the vast related literature (e.g., Grossman 1981, Shavell 1994, Guo and Zhao 2009, Board 2009, Ghosh and Galbreth 2013, Guan and Chen 2017, Li and Peeters 2017, Iyer and Singh 2018), we assume that the disclosed product quality information is truthful and the manufacturer's marginal cost of production is constant and normalized to zero. The firms' payoffs and the consumer's utility are zero if no trade takes place. Because the game includes multiple rounds of strategic interactions between the manufacturer and the retailer, backward induction is applied to ensure subgame perfection throughout this study.

\section{Equilibrium Analysis and Results}

In this section, we first investigate the manufacturer's equilibrium product quality information acquisition and disclosure strategies in a decentralized supply chain under voluntary disclosure of product quality information and mandatory disclosure of product quality information, respectively. We then compare the equilibrium strategies and payoffs of the firms (i.e., the manufacturer and the retailer) and the consumer surplus under voluntary disclosure and mandatory disclosure.

\subsection{Voluntary Disclosure}

We first derive the firms' equilibrium pricing strategies given the manufacturer's quality information acquisition and disclosure decisions. There are three subgames, depending on the information acquisition and disclosure decisions $\left(s_{A}, s_{D}\right) \in(a, d ; a, n d ; n a, n d) .{ }^{11}$ We then start the analysis by assuming that the manufacturer has acquired product quality information $\left(s_{A}=a\right)$. Under such a circumstance, if the manufacturer discloses the quality information that he has acquired

\footnotetext{
11 Note that it never happens that $\left(s_{A}, s_{D}\right)=(n a, d)$ given that the manufacturer is prohibited from arbitrarily disclosing quality information.
} 
$\left(s_{D}=d\right)$, in the last stage, given the wholesale price $w$, the retailer's optimal retail price that maximizes her payoff $\tilde{\pi}_{d}=(p-w)(\theta q-p)$, is $\widetilde{p}_{d}=\frac{\theta q+w}{2}$. If the manufacturer does not disclose quality information, the retailer's payoff can be written as $\widetilde{\pi}_{n d}=\int_{q}(p-w)(\theta q-p) d F\left(q \mid s_{D}=n d\right)$, where the subscript $n d$ denotes non-disclosure of quality information. Here, $q_{n d}=E\left[q \mid s_{D}=n d\right]$ denotes updated quality expectations upon non-disclosure of the downstream parties, whose value will be derived later. Thus, the retailer's optimal retail price is $\widetilde{p}_{n d}=\frac{\theta q_{n d}+w}{2}$.

In the third stage, if the manufacturer has acquired product quality information and disclosed it, his payoff, excluding the cost of quality information acquisition, can be written as $\widetilde{\Pi}_{d}=\frac{w(\theta q-w)}{2}$. The first-order condition gives the optimal wholesale price that the manufacturer charges: $\widetilde{w}_{d}=$ $\frac{\theta q}{2}$. Then, the manufacturer's payoff is $\widetilde{\Pi}_{d}=\frac{\theta^{2} q^{2}}{8}$. The quadratic form of the manufacturer's payoff with respect to $q$ shows that there is a value associated with obtaining product quality information. Ignoring the strategic effect of his action on the retailer's demand for the moment, if the manufacturer does not acquire product quality information, he charges a wholesale price based on average quality, and his payoff would be $\frac{\theta^{2}(E[q])^{2}}{8}$. If he has learned the realization of $q$ and disclosed it, his payoff is given by $\frac{\theta^{2} q^{2}}{8}$, the expected value of which is $\frac{\theta^{2}\left[(E[q])^{2}+\sigma^{2}\right]}{8}$, where $\sigma^{2}$ is the variance of $q$. Actually, under voluntary disclosure, the manufacturer is endowed with flexibility to discretionarily disclose the quality information that he has acquired. This subsequently allows the manufacturer to extract more surplus from acquiring product quality information.

Let us consider that in the third stage, the manufacturer has not disclosed quality information. Accordingly, the downstream parties generate an updated quality expectation $q_{n d}$, and the manufacturer's payoff is $\widetilde{\Pi}_{n d}=\frac{w\left(\theta q_{n d}-w\right)}{2}$. The manufacturer's optimal wholesale price is then $\widetilde{w}_{n d}=\frac{\theta q_{n d}}{2}$, and the associated payoff is $\widetilde{\Pi}_{n d}=\frac{\theta^{2}\left(q_{n d}\right)^{2}}{8}$. A comparison between $\widetilde{\Pi}_{d}$ and $\widetilde{\Pi}_{n d}$ gives us the following condition under which quality information disclosure is beneficial.

Lemma 1. Under voluntary disclosure, if the manufacturer has acquired the precise quality information of his product, he discloses the information if and only if $q>q_{n d}=E\left[q \mid s_{D}=n d\right]$.

Suppose that the manufacturer has privately observed the precise quality information of his product. He would then disclose it only if it is profitable to do so. That is, the disclosed quality information must be high enough to improve the downstream parties' quality expectation upon non-disclosure (i.e., $q>q_{n d}$ ). Otherwise, it is more beneficial for the manufacturer to remain silent.

We next characterize the value of $q_{n d}$, a key step in determining the equilibrium strategy. Notably, when downstream parties observe non-disclosure, they cannot confirm whether it is because the manufacturer has not acquired quality information or because the manufacturer has acquired the quality information that is not favorable (i.e., $q \leq q_{n d}$ ). Either of these two cases can result in 
a consequence of non-disclosure. Accordingly, we derive the conditional probability of each case. First, let us use $\alpha(0<\alpha<1)$ to denote the likelihood that the manufacturer acquires quality information after observing quality information acquisition cost. Thus, the probability of not acquiring quality information is $1-\alpha$. Second, if the manufacturer has acquired quality information but still chooses non-disclosure, this implies that the manufacturer's product quality level must be sufficiently low $q \leq q_{n d}$. Thus, it happens with a probability that $\operatorname{Pr}\left(s_{A}=a, q \leq q_{n d}\right)=\alpha F\left(q_{n d}\right)$. By combining these and using Bayes' rule, we can identify the conditional probability for each case, where

$$
\operatorname{Pr}\left(s_{A}=n a \mid s_{D}=n d\right)=\frac{1-\alpha}{(1-\alpha)+\alpha F\left(q_{n d}\right)}, \operatorname{Pr}\left(s_{A}=a \mid s_{D}=n d\right)=\frac{\alpha F\left(q_{n d}\right)}{(1-\alpha)+\alpha F\left(q_{n d}\right)} .
$$

This subsequently leads to the downstream parties' product quality expectation upon non-disclosure, where

$$
q_{n d}=\frac{1-\alpha}{(1-\alpha)+\alpha F\left(q_{n d}\right)} E[q]+\frac{\alpha F\left(q_{n d}\right)}{(1-\alpha)+\alpha F\left(q_{n d}\right)} E\left[q \mid q \leq q_{n d}\right] .
$$

Given that product quality is uniformly distributed between $[0,1], F\left(q_{n d}\right)=q_{n d}, E[q]=\frac{1}{2}$ and $E\left[q \mid q \leq q_{n d}\right]=\frac{q_{n d}}{2}$. Thus, we can derive the equilibrium quality expectation $q_{n d}$

$$
q_{n d}=\frac{\sqrt{1-\alpha}-(1-\alpha)}{\alpha}
$$

where the value hinges on the success rate of quality information acquisition.

It is evident that $q_{n d}$ monotonically decreases in $\alpha$, which implies that the higher the chance of quality information acquisition is for the manufacturer, the more the quality information would be disclosed by the manufacturer. In particular, if $\alpha=1$, indicating that the manufacturer always acquires quality information, in equilibrium, the manufacturer discloses all quality information (i.e., $q_{n d}=0$ ). This result is consistent with unraveling theory. If the downstream parties can confirm that the manufacturer has observed quality information and disclosure is costless, then non-disclosure conveys the worst quality such that, in equilibrium, all quality information should be disclosed. On the other hand, if $\alpha=0$, indicating that the manufacturer is unable to acquire quality information, $q_{n d}$ equals its expected value $E[q]=\frac{1}{2}$ based on equation (1). Thus, we can confirm that, in equilibrium, $q_{n d}$ can never exceed $E[q]$ (i.e., $0<q_{n d}<\frac{1}{2}$ ), where the value is further determined by quality information acquisition rate $\alpha$.

We now proceed to the first stage to identify the manufacturer's quality information acquisition strategy. His payoffs from acquisition and no acquisition are given by $\widetilde{\Pi}_{a}=\int_{0}^{q_{n d}}\left(\widetilde{\Pi}_{n d}\right) d F(q)+$ $\int_{q_{n d}}^{1}\left(\widetilde{\Pi}_{d}\right) d F(q)-k$ and $\widetilde{\Pi}_{n a}=\int_{0}^{1}\left(\widetilde{\Pi}_{n d}\right) d F(q)$, respectively. With $q \sim U(0,1)$, we can obtain that $\widetilde{\Pi}_{a}=\frac{\theta^{2}\left[2\left(q_{n d}\right)^{3}+1\right]}{24}, \widetilde{\Pi}_{n a}=\frac{\theta^{2}\left(q_{n d}\right)^{2}}{8}$, and $\widetilde{\Pi}_{a}-\widetilde{\Pi}_{n a}=\frac{\theta^{2}\left[2\left(q_{n d}\right)^{3}-3\left(q_{n d}\right)^{2}+1\right]}{24}-k$. The manufacturer chooses 
to acquire product quality information only if it is profitable, thus the manufacturer would acquires the precis quality information about his product when $k \leq \widetilde{k}$, where $\widetilde{k}=\frac{\theta^{2}\left[2\left(q_{n d}\right)^{3}-3\left(q_{n d}\right)^{2}+1\right]}{24}$, under voluntary disclosure. Because the quality information acquisition cost $k$ is a random variable, the probability that the manufacturer undertakes quality information acquisition should be $\operatorname{Pr}(k \leq \widetilde{k})=\alpha$. The subsequent equilibrium quality information acquisition and disclosure strategies are listed in Proposition 1.

Proposition 1. Under voluntary disclosure, the manufacturer acquires the precise quality information of his product if and only if $k \leq \widetilde{k}$ and discloses it when $q>q_{n d}$, where $\widetilde{k}=\frac{\theta^{2}\left\{2\left[q_{n d}(\alpha)\right]^{3}-3\left[q_{n d}(\alpha)\right]^{2}+1\right\}}{24}$, and $\alpha$ satisfies the condition that $\alpha=\frac{\theta^{2}\left\{2\left[q_{n d}(\alpha)\right]^{3}-3\left[q_{n d}(\alpha)\right]^{2}+1\right\}}{24 K}$.

In Proposition 1, $\widetilde{k}$ denotes the cutoff value for the quality information acquisition cost, the manufacturer would voluntarily acquire the precise product quality information only if the realized information acquisition cost is lower than this value. As mentioned above, this equilibrium quality information acquisition strategy is driven by strategic interactions between the downstream parties' quality updating process and the manufacturer's quality information disclosure behavior. Since $\alpha=\operatorname{Pr}(k \leq \widetilde{k})=\widetilde{k}, \widetilde{k}=\frac{\theta^{2}\left[2\left(q_{n d}\right)^{3}-3\left(q_{n d}\right)^{2}+1\right]}{24}$ and $q_{n d}=\frac{\sqrt{1-\alpha}-(1-\alpha)}{\alpha}$, it is shown in Appendix A that there is a unique solution to $\alpha$. Consequently, both $q_{n d}$ and $\widetilde{k}$ are well defined. Given that $0<q_{n d}<\frac{1}{2}$ and $\widetilde{k}$ is strictly decreasing in $q_{n d}$ for $q_{n d} \in\left(0, \frac{1}{2}\right)$, the cutoff value $\widetilde{k}$ is in the range $\left(\frac{\theta^{2}}{48}, \frac{\theta^{2}}{24}\right)$ (shown in Figure 2). In the next part, we will further investigate the case in which the manufacturer is mandated to disclose any product quality information when he undertakes quality information acquisition.

\subsection{Mandatory Disclosure}

Under mandatory disclosure, any product quality information acquired by the manufacturer must be disclosed publicly. Therefore, if the manufacturer acquires quality information, then both the retailer and consumers can observe the same quality information as the manufacturer. In the last stage, given the wholesale price $w$, the retailer's optimal retail price that maximizes her payoff is $\hat{p}_{d}=\frac{\theta q+w}{2}$. In the third stage, in anticipation of the retailer's optimal response, the optimal wholesale price set by the manufacturer is $\widehat{w}_{d}=\frac{\theta q}{2}$. On the other hand, if the manufacturer does not acquire quality information, then all players keep the same belief on the product quality $\bar{q}=E[q]=\frac{1}{2}$, thus the retailer's optimal retail price is $\hat{p}_{n d}=\frac{\theta \bar{q}+w}{2}$, and the manufacturer's optimal wholesale price is $\widehat{w}_{n d}=\frac{\theta}{4}$. Then, we can obtain that in the third stage, the manufacturer's optimal payoff (excluding the cost of quality information acquisition) is $\widehat{\Pi}_{d}=\frac{\theta^{2} q^{2}}{8}$ if the manufacturer acquires quality information, and the optimal payoff achieved by the manufacturer 
is $\widehat{\Pi}_{n d}=\frac{\theta^{2} \bar{q}^{2}}{8}$ if the manufacturer does not acquire quality information. Based on above analysis, we move to the first stage to identify the manufacturer's quality information acquisition strategy under mandatory disclosure. As the manufacturer's quality information disclosure strategy is predetermined in the first stage of quality information acquisition, if the manufacturer does acquire quality information, then for each realization of $q$, the payoff is given by $\widehat{\Pi}_{a}=\int_{0}^{1}\left(\widehat{\Pi}_{d}\right) d F(q)-k$, and his payoff is given by $\widehat{\Pi}_{n a}=\int_{0}^{1}\left(\widehat{\Pi}_{n d}\right) d F(q)$ if he does not acquire quality information. Then $\widehat{\Pi}_{a}-\widehat{\Pi}_{n a}=\frac{\theta^{2}}{8} \int_{0}^{1}\left(q^{2}-\bar{q}^{2}\right) d F(q)-k=\frac{\theta^{2} \sigma^{2}}{8}-k$, where $\sigma^{2}$ is the variance of the product quality $q$. As $q \sim U(0,1), \widehat{\Pi}_{a}-\widehat{\Pi}_{n a}=\frac{\theta^{2}}{96}-k$. The manufacturer chooses to acquires product quality information only if it is profitable, thus the manufacturer would acquire the precise quality information about his product if and only if $k \leq \widehat{k}$, where $\widehat{k}=\frac{\theta^{2}}{96}$, under mandatory disclosure. The subsequent equilibrium quality information acquisition strategy are listed in the following proposition.

Proposition 2. Under mandatory disclosure, the manufacturer acquires the precise quality information of his product if and only if $k \leq \widehat{k}=\frac{\theta^{2}}{96}$.

Still, under mandatory disclosure, the equilibrium quality information acquisition strategy exhibits a cutoff structure in which the manufacturer acquires quality information only if $k \leq \widehat{k}$. Recalling Proposition 1, we next examine how the manufacturer's quality information acquisition incentive $(\widetilde{k}$ or $\widehat{k})$ changes with respect to voluntary disclosure of quality information and mandatory disclosure of quality information, whose results and graphical illustrations are shown in Corollary 1 and Figure 2, respectively.

Corollary 1. The manufacturer is more likely to acquire the precise quality information of his product under voluntary disclosure than under mandatory disclosure.

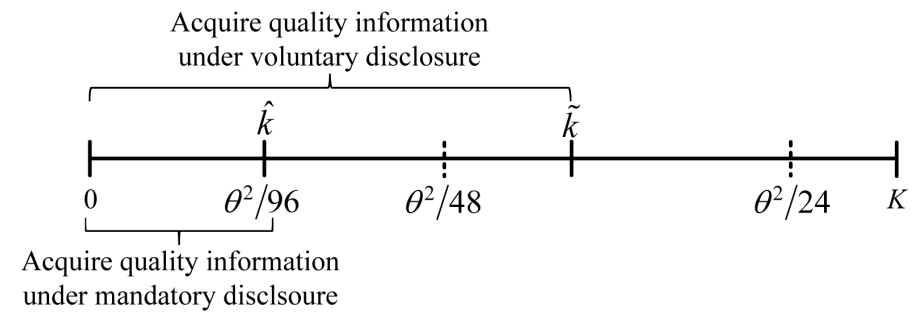

Figure 2 The ranges of $k$ for quality information acquisition

Figure 2 explicitly indicates that the manufacturer has a much stronger quality information acquisition incentive when he can decide whether to disclose the quality information that he has acquired. This situation can be explained as follows: under mandatory disclosure, quality information acquisition helps the manufacturer craft a more precise pricing strategy but does not 
endow him with an information advantage over downstream parties. This is because if the manufacturer has acquired quality information, he must truthfully disclose it. In this sense, quality information acquisition only generates an efficiency effect on the manufacturer's side. Differently, if disclosure is voluntary, then quality information acquisition not only allows the manufacturer to craft a better pricing strategy but also endows him with quality information that is superior to that of downstream parties. As a result, the manufacturer can design a more flexible quality information disclosure strategy in anticipation of downstream parties' quality expectation. Thus, it is evident that the impact of quality information acquisition is more pronounced under voluntary disclosure, and there is a stronger quality information acquisition incentive for the manufacturer under such circumstance.

Corollary 2. The manufacturer is more likely to disclose the quality information of his product under voluntary disclosure than under mandatory disclosure.

Corollary 2 further examines which quality information disclosure system can give rise to a higher extent of quality information transparency in the supply chain. This can be verified by the comparison of manufacturer's disclosure probabilities under voluntary disclosure and mandatory disclosure. For example, under voluntary disclosure, the manufacturer would choose disclosure only if he has acquired the quality information $(k \leq \widetilde{k})$ and the acquired information indicates that the product quality is higher than a threshold $\left(q>q_{n d}\right)$, where $\operatorname{Pr}(k \leq \widetilde{k}) \cdot \operatorname{Pr}\left(q>q_{n d}\right)=\frac{\widetilde{k}\left(1-q_{n d}\right)}{K}$. Under mandatory disclosure, as long as the manufacturer acquires quality information, he must

disclose the information, where $\operatorname{Pr}(k \leq \widehat{k})=\frac{\widehat{k}}{K}$. Given that $q_{n d} \in\left(0, \frac{1}{2}\right)$ and $\widetilde{k}=\frac{\theta^{2}\left[2\left(q_{n d}\right)^{3}-3\left(q_{n d}\right)^{2}+1\right]}{24}$, then $\operatorname{Pr}(k \leq \widetilde{k}) \cdot \operatorname{Pr}\left(q>q_{n d}\right)-\operatorname{Pr}(k \leq \widehat{k})=\frac{\theta^{2}}{24 K}\left\{\left[2\left(q_{n d}\right)^{3}-3\left(q_{n d}\right)^{2}+1\right]\left(1-q_{n d}\right)-\frac{1}{4}\right\}>0$. Thus, it is evident that the manufacturer possesses a higher quality information disclosure incentive under voluntary disclosure, despite that he can discretionarily disclose quality information.

\subsection{Payoff Implications}

Since we have derived the manufacturer's equilibrium quality information acquisition and disclosure strategies in two representative cases, we then examine how different quality information disclosure systems influence the payoffs achieved by the firms (i.e., the manufacturer and the retailer) in supply chain. In particular, we are interested in how firms' interim payoffs (after quality information acquisition cost is realized) and ex-ante payoffs (before quality information acquisition cost is realized) change under these two circumstances. We first compare the firms' interim payoffs.

Voluntary Disclosure. Given the equilibrium strategy reported in Proposition 1, it is evident 
that the firms' interim payoffs could exhibit two patterns with respect to different quality information acquisition options, which are elaborated as follows.

The manufacturer acquires product quality information when $k \in[0, \widetilde{k}]$. His interim payoff, after learning the cost of quality information acquisition, is

$$
\widetilde{\Pi}=\underbrace{\int_{0}^{q_{n d}}\left[\frac{\theta^{2}\left(q_{n d}\right)^{2}}{8}\right] d F(q)}_{\text {Non-disclosure }}+\underbrace{\int_{q_{n d}}^{1}\left(\frac{\theta^{2} q^{2}}{8}\right) d F(q)}_{\text {Disclosure }}-k=\frac{\theta^{2}\left[2\left(q_{n d}\right)^{3}+1\right]}{24}-k,
$$

and the retailer's interim payoff is then

$$
\tilde{\pi}=\underbrace{\int_{0}^{q_{n d}}\left[\frac{\theta^{2}\left(q_{n d}\right)^{2}}{16}\right] d F(q)}_{\text {Non-disclosure }}+\underbrace{\int_{q_{n d}}^{1}\left(\frac{\theta^{2} q^{2}}{16}\right) d F(q)}_{\text {Disclosure }}=\frac{\theta^{2}\left[2\left(q_{n d}\right)^{3}+1\right]}{48} .
$$

On the contrary, when $k \in(\widetilde{k}, K]$, the manufacturer does not acquire product quality information, and his interim payoff is

$$
\widetilde{\Pi}=\frac{\theta^{2}\left(q_{n d}\right)^{2}}{8}
$$

and the retailer's interim payoff is

$$
\widetilde{\pi}=\frac{\theta^{2}\left(q_{n d}\right)^{2}}{16}
$$

Mandatory Disclosure. Similarly, we report the firms' interim payoffs when the quality information acquired by the manufacturer must be disclosed. Specifically, when $k \in[0, \widehat{k}]$, the manufacturer acquires product quality information and discloses it. Then, his interim payoff is

$$
\widehat{\Pi}=\int_{0}^{1}\left(\frac{\theta^{2} q^{2}}{8}\right) d F(q)-k=\frac{\theta^{2}}{24}-k,
$$

and the retailer's interim payoff is

$$
\widehat{\pi}=\int_{0}^{1}\left(\frac{\theta^{2} q^{2}}{16}\right) d F(q)=\frac{\theta^{2}}{48} .
$$

When $k \in(\widehat{k}, K]$, the manufacturer does not acquire product quality information, and his interim payoff is

$$
\widehat{\Pi}=\frac{\theta^{2} \bar{q}^{2}}{8}=\frac{\theta^{2}}{32}
$$

and the retailer's interim payoff is

$$
\widehat{\pi}=\frac{\theta^{2} \bar{q}^{2}}{16}=\frac{\theta^{2}}{64} .
$$

Proposition 3. The manufacturer's interim payoff is higher under voluntary disclosure than that under mandatory disclosure if $k \leq \frac{\theta^{2}\left[8\left(q_{n d}\right)^{3}+1\right]}{96}$ and lower if $k>\frac{\theta^{2}\left[8\left(q_{n d}\right)^{3}+1\right]}{96}$. The retailer's interim payoff is higher under voluntary disclosure than that under mandatory disclosure if $k \leq \widetilde{k}$ and lower if $k>\widetilde{k}$. 

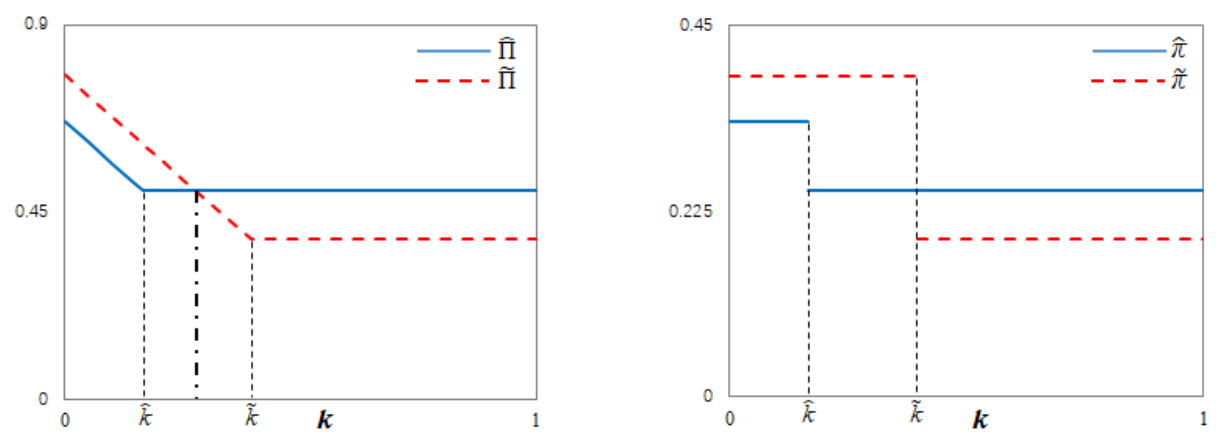

Figure 3 The comparison between the manufacturer's interim payoff, the retailer's interim payoff under voluntary disclosure and those under mandatory disclosure (here, $\theta=4, K=1$ )

A surprising result arises in Proposition 3, which is graphically illustrated in Figure 3. That is, although the manufacturer is more enthusiastic about quality information acquisition under voluntary disclosure, we show that the manufacturer's profitability may be hurt by voluntary disclosure more than by mandatory disclosure. This unintended result arises under the condition that the manufacturer forgoes quality information acquisition under both voluntary disclosure and mandatory disclosure $(k>\widetilde{k})$, or the manufacturer would undertake quality information acquisition under voluntary disclosure when the cost of quality information acquisition is high and forgo quality information acquisition under mandatory disclosure $\left(\frac{\theta^{2}\left[8\left(q_{n d}\right)^{3}+1\right]}{96}<k \leq \widetilde{k}\right)$. The result is rooted in how downstream parties interpret the manufacturer's non-disclosure behaviors under different quality information disclosure systems, which can be explained as follows.

First, when the cost of quality information acquisition is relatively high and it prevents the manufacturer from acquiring the precise quality information about his product under both voluntary disclosure and mandatory disclosure, the outcome of non-disclosure under mandatory disclosure implies that the manufacturer has not acquired quality information, leading to a quality expectation of $\bar{q}$. In contrast, under voluntary disclosure, non-disclosure implies that the manufacturer either does not acquire quality information or has acquired quality information but found that quality is relatively low. This subsequently leads to a quality expectation of $q_{n d}<\bar{q}$. Therefore, under such a circumstance, voluntary disclosure reduces the downstream parties' quality expectation upon non-disclosure in comparison to mandatory disclosure. This reduced quality expectation consequently makes the manufacturer worse off under voluntary disclosure, even though he can better observe quality information compared with downstream parties.

Second, let us consider the case in which the cost of quality information acquisition is relatively low and the manufacturer acquires the precise quality information of his product under both voluntary disclosure and mandatory disclosure. Under mandatory disclosure, the manufacturer has 
to report all quality information, regardless of whether quality is high or low. Thus, from an exante perspective, the expected quality level is still $\bar{q}$. In contrast, under voluntary disclosure, the manufacturer discloses the quality information that he has acquired when $q>q_{n d}$ and conceals the quality information when $q \leq q_{n d}$, but this keeps the downstream parties' quality expectation at $q_{n d}$ because the downstream parties cannot confirm whether the manufacturer's non-disclosure is driven by no quality information acquisition or low quality. Thus, we show that if quality information acquisition cost is low, voluntary disclosure actually improves the downstream parties' quality information in comparison to mandatory disclosure, which leads to a higher payoff for the manufacturer.

Regarding the retailer, we show that she is always better off (worse off) under voluntary disclosure than under mandatory disclosure when the manufacturer undertakes (forgoes) quality information acquisition. The result is driven by the consumer's strategic interpretation of the manufacturer's disclosure behaviors under different quality information disclosure systems. As mentioned above, compared with mandatory disclosure, non-disclosure conveys a higher (lower) quality signal to the consumer under voluntary disclosure when quality information acquisition cost is low (high). Accordingly, the retailer obtains a higher payoff under voluntary disclosure when the manufacturer acquires quality information under both voluntary disclosure and mandatory disclosure (when $k \leq \widehat{k}$ ) but obtains a lower payoff under voluntary disclosure when the manufacturer forgoes quality information acquisition under both voluntary disclosure and mandatory disclosure (when $k>\widetilde{k}$ ). In addition, when the manufacturer chooses quality information acquisition under voluntary disclosure but no quality information acquisition under mandatory disclosure, the surplus from voluntary disclosure becomes apparent to the retailer. This is because in addition to the enhanced quality expectation, the retailer can now better determine her retail price via the manufacturer's voluntary disclosure behavior.

Finally, we check how the firms' ex-ante payoffs change with respect to voluntary disclosure of quality information and mandatory disclosure information. Under voluntary disclosure, as the manufacturer's interim payoff is given by expression (2) if $k \leq \widetilde{k}$ and by expression (4) if $\widetilde{k}<k \leq K$, the manufacturer's ex-ante payoff can be expressed as

$$
E[\widetilde{\Pi}]=\underbrace{G(\widetilde{k})\left\{\int_{0}^{q_{n d}}\left[\frac{\theta^{2}\left(q_{n d}\right)^{2}}{8}\right] d F(q)\right\}}_{\text {Acquisition \& Non-disclosure }}+\underbrace{G(\widetilde{k})\left[\int_{q_{n d}}^{1}\left(\frac{\theta^{2} q^{2}}{8}\right) d F(q)\right.}_{\text {Acquisition \& Disclosure }}]+\underbrace{[1-G(\widetilde{k})] \cdot \frac{\theta^{2}\left(q_{n d}\right)^{2}}{8}}_{\text {No acquisition }}-\int_{0}^{\widetilde{k}} k d G(k) .
$$

For the retailer, as her interim payoff is given by expression (3) if $k \leq \widetilde{k}$ and by expression (5) if 
$\widetilde{k}<k \leq K$, her ex-ante payoff is

$$
E[\widetilde{\pi}]=\underbrace{G(\widetilde{k})\left\{\int_{0}^{q_{n d}}\left[\frac{\theta^{2}\left(q_{n d}\right)^{2}}{16}\right] d F(q)\right\}}_{\text {Acquisition \& Non-disclosure }}+\underbrace{G(\widetilde{k})\left[\int_{q_{n d}}^{1}\left(\frac{\theta^{2} q^{2}}{16}\right) d F(q)\right]}_{\text {Acquisition \& Disclosure }}+\underbrace{[1-G(\widetilde{k})] \cdot \frac{\theta^{2}\left(q_{n d}\right)^{2}}{16}}_{\text {No acquisition }} .
$$

Under mandatory disclosure, the manufacturer's interim payoff is given by expression (6) if $k \leq \widehat{k}$ and by expression (8) if $\widehat{k}<k \leq K$, and the retailer's interim payoff is given by expression (7) if $k \leq \widehat{k}$ and by expression (9) if $\widehat{k}<k \leq K$. Then, the manufacturer's ex-ante payoff can be written as

$$
E[\widehat{\Pi}]=\underbrace{G(\widehat{k})\left[\int_{0}^{1}\left(\frac{\theta^{2} q^{2}}{8}\right) d F(q)\right]}_{\text {Acquisition }}+\underbrace{[1-G(\widehat{k})] \cdot \frac{\theta^{2}}{32}}_{\text {No acquisition }}-\int_{0}^{\widehat{k}} k d G(k),
$$

and the retailer's ex-ante payoff is

$$
E[\widehat{\pi}]=\underbrace{G(\widehat{k})\left[\int_{0}^{1}\left(\frac{\theta^{2} q^{2}}{16}\right) d F(q)\right]}_{\text {Acquisition }}+\underbrace{[1-G(\widehat{k})] \cdot \frac{\theta^{2}}{64}}_{\text {No acquisition }} .
$$

Comparing the equilibrium ex-ante payoffs achieved by the manufacturer and the retailer under voluntary disclosure and mandatory disclosure yields the following proposition. Detailed proofs are shown in Appendix A.

Proposition 4. The manufacturer's ex-ante payoff is higher under mandatory disclosure, while the retailer's ex-ante payoff is higher under voluntary disclosure.

Interestingly, we show that from an ex-ante perspective, the manufacturer is consistently worse off while the retailer is better off under voluntary disclosure than under mandatory disclosure. Note that a firm's ex-ante payoff is the aggregation of all its interim payoffs under every possible quality information acquisition cost. As we have shown above, from either the manufacturer's or the retailer's perspective, voluntary disclosure can give rise to a higher interim payoff when the information acquisition cost is relatively low but to a lower interim payoff when the information acquisition cost is high in comparison to the mandatory disclosure. This can be explained that when quality information acquisition cost is low, it induces the manufacturer to adopt quality information acquisition, then voluntary disclosure allows the manufacturer to strategically withhold negative quality information (this is absent under mandatory disclosure), which is beneficial to both the manufacturer's profitability and retailer's profitability. In contrast, when quality information acquisition cost is high and prevents the manufacturer from adopting quality information acquisition, then voluntary disclosure of quality information results in a lower quality expectation 
from the consumer's side, and this hurts both the manufacturer and the retailer. Finally, these conflicting outcomes on the firms' interim payoffs form their adverse preferences toward these two quality information disclosure systems.

\subsection{Consumer Surplus}

Based on the above analysis, we further examine how the consumer surplus changes with respect to different quality information acquisition and disclosure options. In particular, under voluntary disclosure, if the manufacturer discloses the product quality information that he has acquired, the consumer surplus is $\widetilde{C S}_{d}=\left(\theta q-\widetilde{p}_{d}\right) \widetilde{Q}_{d}-\frac{1}{2}\left(\widetilde{Q}_{d}\right)^{2}=\frac{\theta^{2} q^{2}}{32}$. Otherwise, the consumer surplus is given by $\widetilde{C S}_{n d}=\left(\theta q_{n d}-\widetilde{p}_{n d}\right) \widetilde{Q}_{n d}-\frac{1}{2}\left(\widetilde{Q}_{n d}\right)^{2}=\frac{\theta^{2}\left(q_{n d}\right)^{2}}{32}$. Thus, we can present the expected consumer surplus under voluntary disclosure, where

$$
\widetilde{E C S}=\underbrace{G(\widetilde{k})\left\{\int_{0}^{q_{n d}}\left[\frac{\theta^{2}\left(q_{n d}\right)^{2}}{32}\right] d F(q)\right\}}_{\text {Acquisition \& Non-disclosure }}+\underbrace{G(\widetilde{k})\left[\int_{q_{n d}}^{1}\left(\frac{\theta^{2} q^{2}}{32}\right) d F(q)\right]}_{\text {Acquisition \& Disclosure }}+\underbrace{[1-G(\widetilde{k})] \cdot \frac{\theta^{2}\left(q_{n d}\right)^{2}}{32}}_{\text {No acquisition }} .
$$

Under mandatory disclosure, if the manufacturer acquires the precise quality information of his product, the consumer surplus is $\widehat{C S}_{d}=\left(\theta q-\widehat{p}_{d}\right) \widehat{Q}_{d}-\frac{1}{2}\left(\widehat{Q}_{d}\right)^{2}=\frac{\theta^{2} q^{2}}{32}$ given that the manufacturer must disclose all quality information. If the manufacturer does not acquire quality information, the consumer surplus is $\widehat{C S}_{n d}=\left(\theta \bar{q}-\widehat{p}_{n d}\right) \widehat{Q}_{n d}-\frac{1}{2}\left(\widehat{Q}_{n d}\right)^{2}=\frac{\theta^{2} \bar{q}^{2}}{32}$. Then, the expected consumer surplus under mandatory disclosure can be expressed as

$$
\widehat{E C S}=\underbrace{G(\widehat{k})\left[\int_{0}^{1}\left(\frac{\theta^{2} q^{2}}{32}\right) d F(q)\right]}_{\text {Acquisition }}+\underbrace{[1-G(\widehat{k})] \cdot \frac{\theta^{2} \bar{q}^{2}}{32}}_{\text {No acquisition }} .
$$

By comparing the expected consumer surplus under voluntary disclosure and mandatory disclosure, we can obtain the following proposition. Detailed proofs are shown in Appendix A.

Proposition 5. The expected consumer surplus under voluntary disclosure is higher than that under mandatory disclosure.

It is interesting to see that the consumer is better off under voluntary disclosure than under mandatory disclosure. Intuitively, one may think that mandatory disclosure can better protect consumer interests by preventing the manufacturer from withholding product quality information from consumers. Nonetheless, this argument does not hold in a setting in which the manufacturer has to first acquire product quality information by himself. Although voluntary disclosure endows the manufacturer with an information advantage over consumers, it also incentivizes the 
manufacturer to acquire quality information more aggressively. However, under mandatory disclosure, the manufacturer less actively undertakes quality information acquisition, anticipating that any quality information acquired must be truthfully disclosed to consumers.

Based on the above analysis, a key driver of consumer surplus is how likely the consumer is to receive product quality information under voluntary disclosure and mandatory disclosure. Specifically, mandatory disclosure eliminates the manufacturer's private quality information and weakens the manufacturer's quality information acquisition incentive. Voluntary disclosure induces the manufacturer's quality information acquisition but allows the manufacturer to withhold the quality information that he has acquired. These conflicting effects jointly affect the probability that the consumer can receive product quality information under these two quality information disclosure systems. As shown in Corollary 2, voluntary disclosure gives rise to a higher transparency level of product quality information, which is certainly more beneficial to the consumer in comparison to mandatory disclosure.

\section{Discussions}

In this section, we first investigate a case in which the manufacturer might ex-ante know the quality information of his product with some probability. Second, we consider a more general form of demand function. Third, we examine several changes in the distributions of product quality and quality information acquisition cost to check the robustness of our results. Finally, we study an alternative case in which the manufacturer is mandated to acquire product quality information.

\subsection{Ex-ante Informed Manufacturer}

In the basic model, we assume that all the parties including the manufacturer do not know the precise quality information of his product ex-ante, a setting that has been frequently adopted by the prior literature such as Matthews and Postlewaite (1985), Shavell (1994), Arya et al. (2014), Yehezkel (2014), Dahm et al. $(2009,2016)$. Nonetheless, it is also reasonable to assume that the upstream manufacturer, who is involved in the production process, may privately possess some superior knowledge about his product quality. To this end, this subsection investigates a new case wherein the manufacturer can be informed about product quality information with a probability $\rho$ ex-ante and be uninformed of quality information with a probability $1-\rho$ ex-ante, where $\rho \in$ $(0,1)$. When $\rho=0$, it degenerates to our basic model; when $\rho=1$, the manufacturer fully knows 
product quality information ex-ante, then it goes back to the unraveling model (Grossman 1981, Milgrom 1981), in which the manufacturer would choose to fully disclose quality information (i.e., the classic rule of "unraveling"). The downstream parties (i.e., the retailer and the consumers) know such a probability $\rho$ but keep the prior belief that the product quality follows a uniform distribution that $q \sim U[0,1]$. In this sense, the manufacturer still has the incentive to first acquire the precise product quality information and then disclose it to the downstream parties, so as to derive more surplus. We also consider two quality information disclosure systems: voluntary disclosure and mandatory disclosure, and compare how the firms' performances change with respect to different quality information disclosure systems.

The decision sequences are now as follows. First, if the manufacturer does not know product quality information ex-ante, he needs to decide whether to acquire the precise quality information of his product by incurring a quality information acquisition cost $k$. Still, $k$ is the manufacturer's private information that follows a uniform distribution between $[0, K]$. While if the manufacturer already knows product quality information, he does not need to make the information acquisition decision in this stage. Second, depending on product quality information disclosure system, the manufacturer who has acquired quality information (who already knows quality information) decides whether to disclose the information. Third, the manufacturer sets the wholesale price for each unit of product. Fourth, both the retailer and the consumer update their quality expectation and the retailer charges the retail price.

Voluntary Disclosure. We start from the case in which the disclosure of product quality information is voluntary. Similar to our basic model, we define the probability that an ex-ante uninformed manufacturer acquires the precise quality information of his product as $\alpha$, whose value will be derived later. The downstream parties can rationally infer such a probability of quality information acquisition $\alpha$, and based on it, they can further update the quality expectation after observing the manufacturer's subsequent disclosure behavior. If the downstream parties observe non-disclosure, they cannot confirm whether this is because (1) the ex-ante informed manufacturer does not want to disclose quality information due to the quality level is relatively low (i.e., $\left.q \leq q_{1}\right)$; (2) the ex-ante uniformed manufacturer has acquired quality information and found that the information indicates that the quality level is low (i.e., $q \leq q_{2}$ ); or (3) the ex-ante uniformed manufacturer does not acquire quality information. Note that $q_{1}\left(q_{2}\right)$ represents the quality information disclosure cutoff point for the ex-ante informed (uninformed) manufacturer who is indifferent between disclosure and non-disclosure. Let us define the downstream parties' quality expectation upon non-disclosure as $\widetilde{q}_{n d}$, this leads to the equilibrium condition that 


$$
\begin{aligned}
q_{n d}= & \underbrace{\frac{\rho F\left(q_{1}\right)}{\rho F\left(q_{1}\right)+(1-\rho)\left[\alpha F\left(q_{2}\right)+(1-\alpha)\right]} E\left[q_{1} \mid q \leq q_{1}\right]}_{\text {Ex-ante informed \& Non-disclosure }}+\underbrace{\frac{(1-\rho) \alpha F\left(q_{2}\right)}{\rho F\left(q_{1}\right)+(1-\rho)\left[\alpha F\left(q_{2}\right)+(1-\alpha)\right]} E\left[q_{2} \mid q \leq q_{2}\right]}_{\text {Ex-ante uninformed \& Acquisition \& Non-disclosure }} \\
& +\underbrace{\frac{(1-\rho)(1-\alpha)}{\rho F\left(q_{1}\right)+(1-\rho)\left[\alpha F\left(q_{2}\right)+(1-\alpha)\right]} E[q] .}_{\text {Ex-ante uninformed \& No acquisition }}
\end{aligned}
$$

Given that product quality $q$ is uniformly distributed between $[0,1], F\left(q_{1}\right)=q_{1}, F\left(q_{2}\right)=q_{2}$, $E\left[q \mid q \leq q_{1}\right]=\frac{q_{1}}{2}, E\left[q \mid q \leq q_{2}\right]=\frac{q_{2}}{2}$ and $E[q]=\frac{1}{2}$. As the ex-ante informed (uniformed) manufacturer is indifferent between disclosure and nondisclosure at $q_{1}\left(q_{2}\right)$, it leads to the equilibrium condition that $q_{1}=q_{2}=\widetilde{q}_{n d}$. Then, we can identify that in equilibrium,

$$
\widetilde{q}_{n d}=\frac{\sqrt{1-[(1-\rho) \alpha+\rho]}-\{1-[(1-\rho) \alpha+\rho]\}}{(1-\rho) \alpha+\rho},
$$

where $\widetilde{q}_{n d}$ is decreasing in $\rho$ for $\rho \in(0,1)$. That is if the manufacturer has a higher chance to know quality information ex-ante, then he would disclose more quality information. Particularly, when $\rho=1$, the manufacturer is fully informed about product quality information ex-ante, in equilibrium, the manufacturer discloses all quality information (i.e., $\widetilde{q}_{n d}=0$ ). That is consistent with unraveling rule proposed by Grossman (1981) and Milgrom (1981). This can be explained by the fact that when the manufacturer has a higher chance to observe quality information ex-ante, non-disclosure conveys a lower quality expectation to the uninformed retailer and consumers.

We move to the first stage to identify the ex-ante uninformed manufacturer's equilibrium quality information acquisition strategy. Note that the manufacturer's information acquisition strategy should exhibit a cutoff structure, wherein he acquires quality information only if the quality information acquisition cost $k$ is below a threshold $\widetilde{k}$. The manufacturer is indifferent between acquisition and no acquisition at $\widetilde{k}$, and this allows us to derive the manufacturer's equilibrium quality information acquisition strategy, which is shown in the following proposition. Detailed proofs are shown in Appendix A.

Proposition 6. Under voluntary disclosure where the manufacturer is ex-ante informed about product quality information with probability $\rho$, in equilibrium, the ex-ante uninformed manufacturer acquires the precise quality information of his product if and only if $k \leq \widetilde{k}$ and discloses it when $q>\widetilde{q}_{n d}$, where

$$
\widetilde{k}=\frac{\theta^{2}\left[2\left(\widetilde{q}_{n d}\right)^{3}-3\left(\widetilde{q}_{n d}\right)^{2}+1\right]}{24} \text { and } \quad \widetilde{q}_{n d}=\frac{\sqrt{1-[(1-\rho) \alpha+\rho]}-\{1-[(1-\rho) \alpha+\rho]\}}{(1-\rho) \alpha+\rho} \text {. }
$$

We can further show that $\frac{\partial \widetilde{k}}{\partial \rho}>0$, which indicates that the ex-ante uninformed manufacturer would possess a stronger incentive to acquire the precise product quality information of his product when the manufacturer has a higher chance to observe quality information ex-ante. This can be 
explained by considering that when the manufacturer is more likely to know product quality information ex-ante, non-disclosure would convey a lower quality signal to the downstream parties. This reversely pushes the ex-ante uninformed manufacturer to undertake quality information acquisition and to disclose the information once it indicates the product quality is high. Otherwise, the ex-ante uninformed manufacturer cannot claim any quality information to the downstream parties.

Mandatory Disclosure. Under mandatory disclosure, if the manufacturer, who is ex-ante uninformed about the quality information of his product, chooses to acquire the precise quality information, he must disclose the acquired quality information. Therefore, if the downstream parties observe non-disclosure, there are two possibilities: (1) the manufacturer is ex-ante informed about product quality information but does not want to disclose the information due to the information indicates the product quality is relatively low (i.e., $q \leq q_{3}$ ); (2) the manufacturer is ex-ante uninformed and does not acquire quality information. This subsequently leads to the downstream parties' quality expectation upon manufacturer's non-disclosure,

$$
\widehat{q}_{n d}=\underbrace{\frac{\rho F\left(q_{3}\right)}{\rho F\left(q_{3}\right)+(1-\rho)} E\left[q \mid q \leq q_{3}\right]}_{\text {Ex-ante informed \& Non-disclosure }}+\underbrace{\frac{(1-\rho)}{\rho F\left(q_{3}\right)+(1-\rho)} E[q]}_{\text {Ex-ante uninformed \& No acquisition }} .
$$

Given that product quality $q$ is uniformly distributed between $[0,1], F\left(q_{3}\right)=q_{3}, E\left[q \mid q \leq q_{3}\right]=\frac{q_{3}}{2}$ and $E[q]=\frac{1}{2}$. Because the ex-ante informed manufacturer is indifferent between disclosure and nondisclosure at $q_{3}$, it leads to the equilibrium condition that $q_{3}=\widehat{q}_{n d}$. Thus, we can derive the equilibrium quality expectation

$$
\widehat{q}_{n d}=\frac{\sqrt{1-\rho}-(1-\rho)}{\rho} .
$$

It is evident that $\widehat{q}_{n d}$ monotonically decreases in $\rho$ for $\rho \in(0,1)$, which implies that when the manufacturer has a higher chance to observe product quality information ex-ante, he would disclose more quality information. In particular, if $\rho=1$, indicating that the manufacturer is fully informed about product quality information ex-ante, in equilibrium, the manufacturer discloses all quality information (i.e., $\widehat{q}_{n d}=0$ ), which is consistent with unraveling theory. Similar to the above voluntary quality information acquisition case, we can identify the ex-ante uninformed manufacturer's equilibrium quality information acquisition strategy under mandatory disclosure. Because the derivation process is routine, we present the result directly in the following proposition. Detailed proofs are shown in Appendix A.

Proposition 7. Under mandatory disclosure where the manufacturer is ex-ante informed about product quality information with probability $\rho$, in equilibrium, the ex-ante uninformed manufacturer acquires the 
precise quality information of his product if and only if $k \leq \widehat{k}$ and discloses it when $q>\widehat{q}_{n d}$, where

$$
\widehat{k}=\frac{\theta^{2}\left[1-3\left(\widehat{q}_{n d}\right)^{2}\right]}{24} \quad \text { and } \quad \widehat{q}_{n d}=\frac{\sqrt{1-\rho}-(1-\rho)}{\rho} .
$$

Still, we show that $\frac{\partial \hat{k}}{\partial \rho}>0$, indicating that the ex-ante uninformed manufacturer would adopt quality information acquisition more actively when the probability $\rho$ increases. This situation shares a similar principle as that under voluntary disclosure. Moreover, we examine how the quality information acquisition incentive of the ex-ante uninformed manufacturer $(\widetilde{k}$ or $\widehat{k})$ changes under different quality information disclosure systems when the manufacturer knows product quality information with a probability $\rho$ ex-ante. As shown in Figure 4, the ex-ante uniformed manufacturer still has more incentive to acquire the precise quality information of his product under voluntary disclosure (i.e., $\widetilde{k} \geq \widehat{k}$ ), while the gap between $\widetilde{k}$ and $\widehat{k}$ (i.e., $\widetilde{k}-\widehat{k}$ ) will be narrowed when $\rho$ increases.

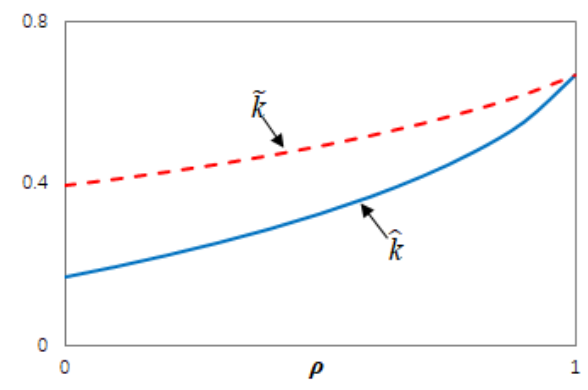

Figure 4 The values of $\widetilde{k}$ and $\widehat{k}$ varying with $\rho$ (here, $\theta=4, K=1$ )

Building upon the manufacturer's equilibrium strategies under voluntary disclosure and mandatory disclosure, the equilibrium ex-ante payoffs of the firms and the expected consumer surplus can be further derived. Under voluntary disclosure, the manufacturer's ex-ante payoff can be expressed as $E[\widetilde{\Pi}]=\rho\left\{\frac{\theta^{2}\left[2\left(\widetilde{q}_{n d}\right)^{3}+1\right]}{24}\right\}+(1-\rho)\left[\frac{\widetilde{k}^{2}}{2 K}+\frac{\theta^{2}\left(\widetilde{q}_{n d}\right)^{2}}{8}\right]$, the retailer's ex-ante payoff is $E[\widetilde{\pi}]=\rho\left\{\frac{\theta^{2}\left[2\left(\widetilde{q}_{n d}\right)^{3}+1\right]}{48}\right\}+(1-\rho)\left[\frac{\widetilde{k}^{2}}{2 K}+\frac{\theta^{2}\left(\widetilde{q}_{n d}\right)^{2}}{16}\right]$, and the expected consumer surplus is $\widetilde{E C S}=$ $\rho\left\{\frac{\theta^{2}\left[2\left(\widetilde{q}_{n d}\right)^{3}+1\right]}{96}\right\}+(1-\rho)\left[\frac{\widetilde{k}^{2}}{4 K}+\frac{\theta^{2}\left(\widetilde{q}_{n d}\right)^{2}}{32}\right]$. Under mandatory disclosure, the manufacturer's ex-ante payoff can be written as $E[\widehat{\Pi}]=\rho\left\{\frac{\theta^{2}\left[2\left(\widehat{q}_{n d}\right)^{3}+1\right]}{24}\right\}+(1-\rho)\left[\frac{\widehat{k}^{2}}{2 K}+\frac{\theta^{2}\left(\widehat{q}_{n d}\right)^{2}}{8}\right]$, the retailer's ex-ante payoff is $E[\widehat{\pi}]=\rho\left\{\frac{\theta^{2}\left[2\left(\widehat{q}_{n d}\right)^{3}+1\right]}{48}\right\}+(1-\rho)\left[\frac{\widehat{k}^{2}}{2 K}+\frac{\theta^{2}\left(\widehat{q}_{n d}\right)^{2}}{16}\right]$, and the expected consumer surplus is $\widehat{E C S}=\rho\left\{\frac{\theta^{2}\left[2\left(\widehat{q}_{n d}\right)^{3}+1\right]}{96}\right\}+(1-\rho)\left[\frac{\widehat{k}^{2}}{4 K}+\frac{\theta^{2}\left(\widehat{q}_{n d}\right)^{2}}{32}\right]$. Then, we can further examine how the firms' exante payoffs and the expected consumer surplus change with respect to voluntary disclosure and mandatory disclosure.

Based on the numerical analysis results shown in Figure 5, one can see that the manufacturer achieves a higher ex-ante payoff under mandatory disclosure, despite that the gap between the 
manufacturer's ex-ante payoff under mandatory disclosure and that under voluntary disclosure (i.e., $E[\widehat{\Pi}]-E[\widetilde{\Pi}]$ ) decreases in $\rho$. This result is consistent with our basic model, because although voluntary disclosure endows the manufacturer with more flexibility in crafting his quality information disclosure strategy, it also results in a lower quality expectation from the retailer and the consumers when the manufacturer does not acquire quality information. However, from the perspectives of the retailer and the consumers, they no longer prefer voluntary disclosure over mandatory disclosure, whose result is further dependent on the magnitude of $\rho$. Although the retailer's ex-ante payoff and the expected consumer surplus are still higher under voluntary disclosure when $\rho$ is low, the retailer's ex-ante payoff and the expected consumer surplus can become higher under mandatory disclosure when $\rho$ increases. Interestingly, we identify a U-shaped relationship between the gaps of voluntary disclosure and mandatory disclosure (i.e., $E[\widetilde{\pi}]-E[\widehat{\pi}]$ and $\widetilde{E C S}-\widehat{E C S}$ ) and the ex-ante informed probability $\rho$.
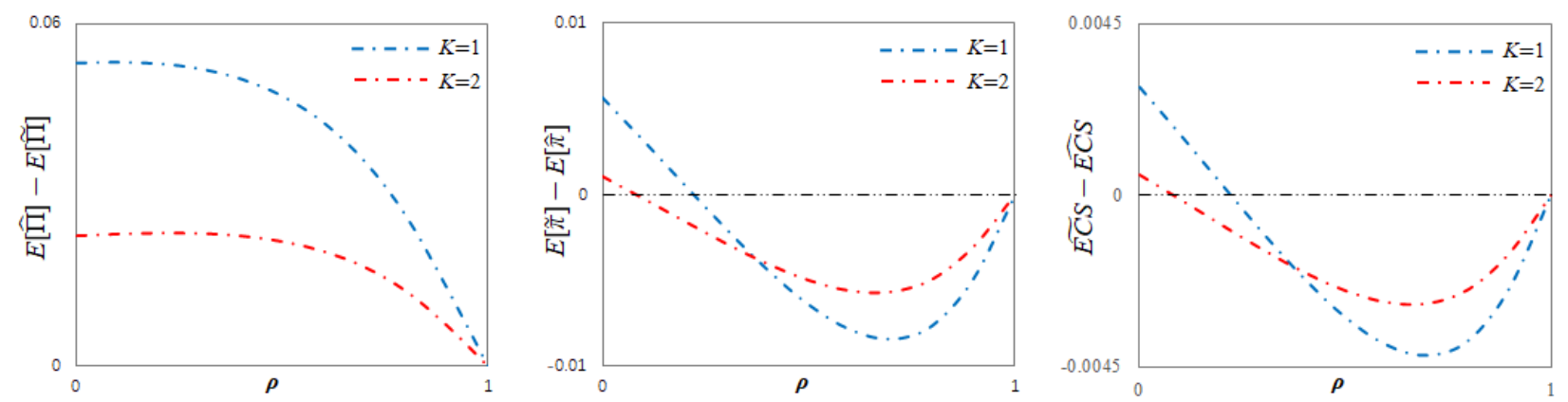

Figure 5 The gaps between the manufacturer's payoff, the retailer's ex-ante payoff, the expected consumer surplus under mandatory disclosure and those under voluntary disclosure varying with $\rho$ (here, $\theta=4$ )

\subsection{Demand Function}

In the basic model, the demand function we adopted is $Q=\theta q-p$, which is derived from the consumer's quadratic utility function: $U=(\theta q) Q-\frac{1}{2} Q^{2}-p Q$ (Singh and Vives, 1984). In this subsection, we further consider a more general form of demand function: $Q=A+\theta q-p$, where $A(>0)$ is a constant value representing the base demand, $\theta$ denotes the consumer's preference for product quality, $q$ is the product quality, and $p$ is the retail price. We will examine whether the main results still hold with this modification. Other settings remain unchanged as that in our basic model.

Note that the change of demand function form mainly changes the equilibrium pricing strategies of the manufacturer and the retailer, while the quality updating process remains essentially similar to that in the basic model. Therefore, we can still derive the manufacturer's equilibrium 
quality information acquisition and disclosure strategies with a standard backward induction, and compare the manufacturer's quality information acquisition incentives under voluntary disclosure and mandatory disclosure. Since such a derivation process is routine and tedious, we then present the results in the following proposition directly and leave the related proofs to Appendix B in the Supplement File.

Proposition 8. With the change of demand function form, in equilibrium:

(1) Under voluntary disclosure, the manufacturer acquires the precise quality information of his product if and only if $k \leq \widetilde{k}$ and disclose it when $q>q_{n d}$, where $\widetilde{k}=\frac{\theta^{2}\left[2\left(q_{n d}\right)^{3}-3\left(q_{n d}\right)^{2}+1\right]+3 A \theta\left(1-q_{n d}\right)^{2}}{24}$, $q_{n d}=\frac{\sqrt{1-\alpha}-(1-\alpha)}{\alpha}$ and $\alpha=\operatorname{Pr}(k \leq \widetilde{k})=\frac{\widetilde{k}}{K}$;

(2) Under mandatory disclosure, the manufacturer acquires the precise quality information of his product if and only if $k \leq \widehat{k}$, where $\widehat{k}=\frac{\theta^{2}}{96}$;

(3) The manufacturer has more incentive to acquire the precise quality information of his product under voluntary disclosure than under mandatory disclosure (i.e., $\widetilde{k}>\widehat{k}$ ).

Similar to our basic model, we further compare how the firms' ex-ante payoffs change with respect to different quality information disclosure systems. The results are illustrated in Figure 6. Thus, it shows that the manufacturer's ex-ante payoff is higher under mandatory disclosure, while the retailer's ex-ante payoff is higher under voluntary disclosure. All these results are consistent with the observation in our basic model, indicating that the main insights remain robust with the change of the demand function.
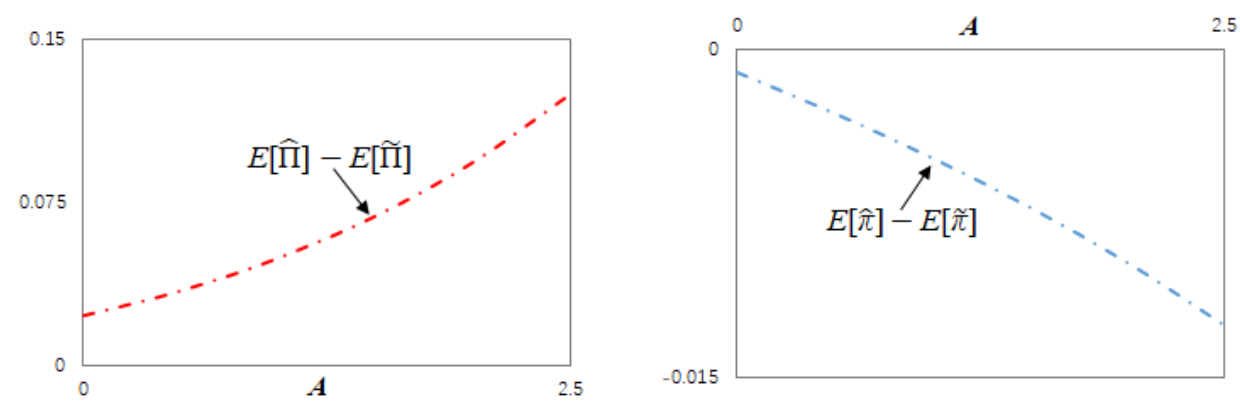

Figure 6 The gaps between the manufacturer's ex-ante payoff, the retailer's ex-ante payoff under mandatory disclosure and those under voluntary disclosure varying with $A$ (here, $A \in[0,2.5], \theta=4, K=2$ ) 


\subsection{Distribution Support}

In this subsection, we first consider a more general distribution of product quality. That is, we assume that the product quality $q=\bar{q}+\varepsilon$, where $\bar{q}(>0)$ is the expected product quality and $\varepsilon$ captures the magnitude of quality variance that can be observed via information acquisition. Moreover, $\varepsilon$ follows a uniform distribution between $[-\delta, \delta]$ (i.e., $\varepsilon \sim U(-\delta, \delta)$ ). A larger $\delta(>0)$ indicates a higher extent of quality variance. $\bar{q}-\delta \geq 0$ ensures that the realized product quality can never be negative. ${ }^{12}$ Other settings remain the same as our basic model.
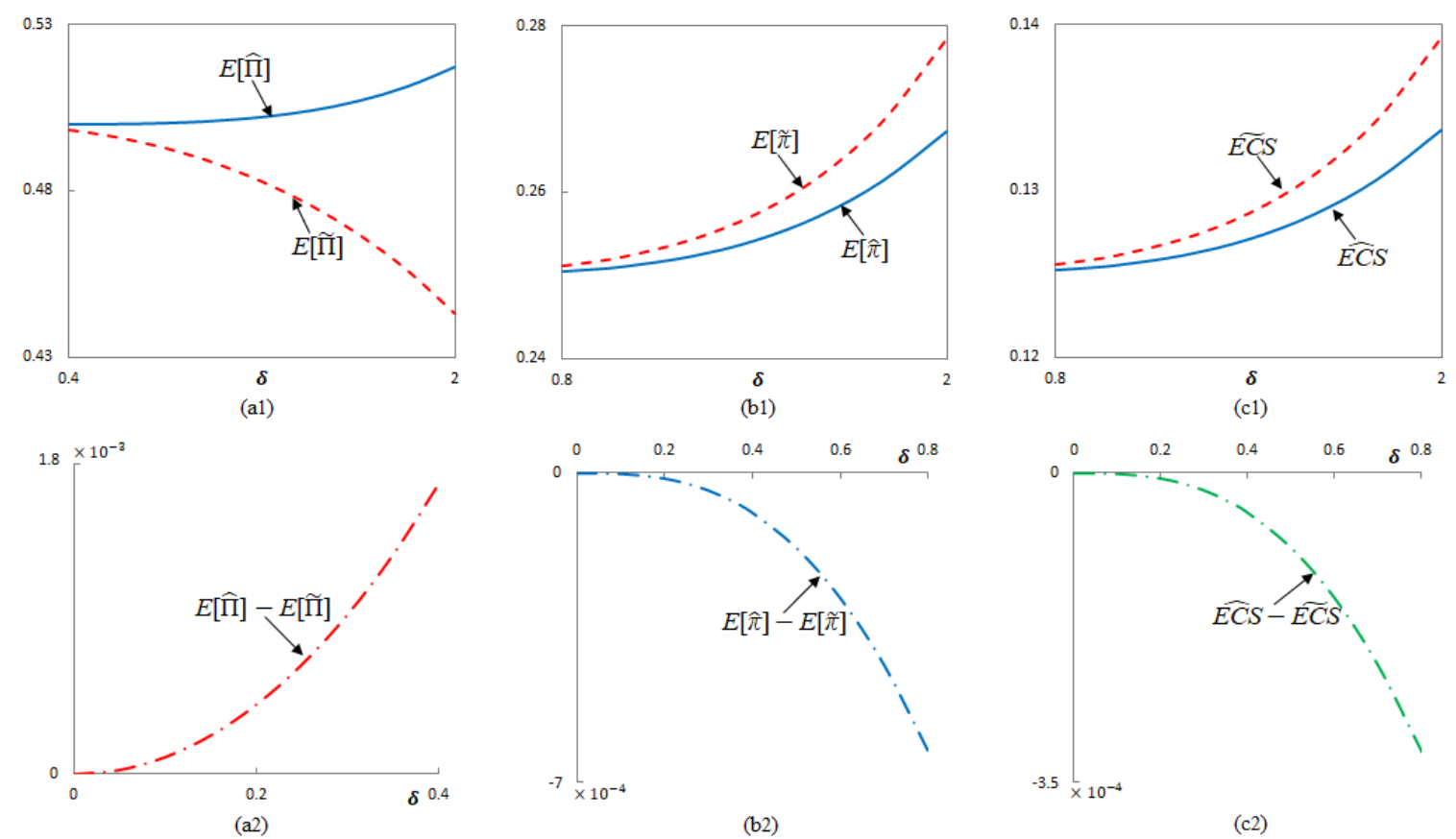

(b2)

(c2)

Figure 7 The impacts of $\delta$ on the manufacturer's ex-ante payoff (left subfigures (a1) and (a2)), the retailer's ex-ante payoff (middle subfigures (b1) and (b2)) and the expected consumer surplus (right subfigures (c1) and (c2)) (here, $\bar{q}=2, \theta=1, K=0.8$ )

One can confirm that all the results in the basic model still hold with this modification, whose detailed derivation process is shown in Appendix B in the Supplement File. For example, the manufacturer is still more likely to acquire product quality information under voluntary disclosure, while his ex-ante payoff is higher under mandatory disclosure. Both the retailer's ex-ante payoff and the expected consumer surplus are higher under voluntary disclosure. We also examine the impact of quality variance $\delta$ on the firms' ex-ante payoffs and the expected consumer

\footnotetext{
${ }^{12}$ This situation is identical to the situation under which the product quality $q$ is on some interval $[a, b](b>a \geq 0)$ and follows a uniform distribution $q \sim U(a, b)$ if $a$ is set equal to $\bar{q}-\delta$ and $b$ is set equal to $\bar{q}+\delta$. and the results under these situations are the same.
} 
surplus. The results are derived via numerical analysis and illustrated in Figure 7. Moreover, one can see that when $\delta=0$, the two cases (i.e., voluntary disclosure and mandatory disclosure) are the same; when $\delta>0$, the two cases become different, and the differences between these two cases become more significant when $\delta$ increases (see subfigures (a2), (b2) and (c2)). Interestingly, under mandatory disclosure, the manufacturer's ex-ante payoff, the retailer's ex-ante payoff and the expected consumer surplus are all increasing in $\delta$; in contrast, under voluntary disclosure, the manufacturer's ex-ante payoff is decreasing in $\delta$, but the retailer's ex-ante payoff and the expected consumer surplus are increasing in $\delta$. Again, these results highlight the significant differences between voluntary disclosure and mandatory disclosure.
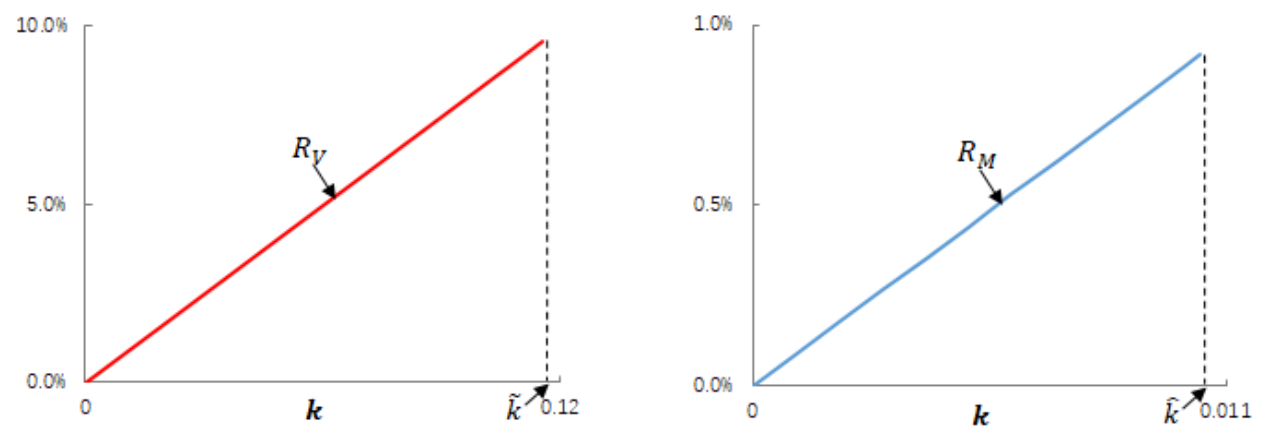

Figure 8 The ratios of $R_{V}$ and $R_{M}$ varying with $k$ (here, $q \sim U(2.5,3.5), \theta=1, K=0.4$ )

Given this more general distribution of product quality, we further provide a sensitivity analysis on how costly the quality information acquisition is in comparison to the manufacturer's payoff under voluntary disclosure and mandatory disclosure. In particular, we define $R_{V}$ as the ratio between the quality information acquisition cost and the manufacturer's expected payoff under voluntary disclosure, and define $R_{M}$ as the ratio between the quality information acquisition cost and the the manufacturer's expected payoff under mandatory disclosure. The results are graphically illustrated in Figure 8 , in which we assume that the product quality $q$ follows a uniform distribution between $[2.5,3.5], \theta=1, K=0.4$, and the horizontal line is the possible cost of quality information acquisition pertaining to the manufacturer adopting quality information acquisition under the two quality information disclosure systems. More details are shown in Appendix B in the Supplement File. Thus, it is evident that under either voluntary disclosure or mandatory disclosure, the ratio can be relatively low, as shown in Figure $8,0 \leq R_{V}<10 \%$ and $0 \leq R_{M}<1 \%$. Actually, as aforementioned, in practice, the quality information acquisition process could be costly depending on the information acquisition methods adopted by the manufacturer. In our motivating example, Golden State Foods has invested heavily in an IoT system to detect and monitor its food quality, and Foxconn has used costly X-ray systems in its assem- 
bly lines to inspect the quality of products. In this sense, we can claim that our analytical results can be carried over into practice, guiding the manufacturer to make more precise product quality information acquisition and disclosure strategies.

Next, we further consider the case wherein the product quality $q$ is continuously distributed on the interval $[a, b]$ with density function $\underline{f}(\cdot)$, distribution function $\underline{F}(\cdot)$, mean $E[q]=\bar{q}$, and variance $\operatorname{Var}[q]=\sigma^{2}$, where $b>a \geq 0$. With this modification, we can demonstrate that the manufacturer still has more incentive to acquire the precise quality information of his product under voluntary disclosure than under mandatory disclosure. In addition, we consider a twopoint distribution of quality information acquisition cost. That is, the manufacturer's information acquisition cost has two possible values $k \in\left\{k_{l}, k_{h}\right\}$, where $k_{h}$ is prohibitively high and $k_{l}$ is negligible, and these two information acquisition cost states occur with ex-ante probabilities of $\operatorname{Pr}\left(k=k_{l}\right)=\beta$ and $\operatorname{Pr}\left(k=k_{h}\right)=1-\beta$, where $\beta \in(0,1)$. As a result, the manufacturer may choose to acquire the precise quality information about his product (if doing so is beneficial) if $k_{l}$ is realized, while he would not acquire such quality information if $k_{h}$ is realized. Even with this modification, we demonstrate that the manufacturer's ex-ante payoff under mandatory disclosure are higher than that under voluntary disclosure, while the retailer's ex-ante payoff and expected consumer surplus are lower under voluntary disclosure. In this sense, we can confirm that the main results are qualitatively similar to those of the basic model. Moreover, it is noteworthy that we investigate the case in which the product quality $q$ is stochastic and takes two possible values $q \in\left\{q_{l}, q_{h}\right\}$, and we find that the main results are still similar to those obtained from our basic model. All the detailed proofs are shown in Appendix B in the Supplement File.

\subsection{Mandatory Acquisition}

In the basic model, we allow for the manufacturer to voluntarily decide whether to acquire the precise quality information of his product and then to disclose that information according to the prevailing quality information disclosure systems. Nonetheless, in practice, quality information acquisition and disclosure are sometimes mandatorily required. For example, all products sold in the EU must have a Restriction of Hazardous Substances (RoHS) certification. ${ }^{13}$ Therefore, in this subsection, we consider a case with mandatory acquisition and mandatory disclosure and compare it with our basic model.

Let us assume that the manufacturer must acquire the precise quality information about his product, and the acquired information is publicly released. Under this circumstance, the manufac-

\footnotetext{
${ }^{13} \mathrm{http}: / /$ ec.europa.eu/environment/waste/rohs_eee/index_en.htm.
} 
turer's equilibrium interim payoff is $\bar{\Pi}=\int_{0}^{1}\left[\frac{\theta^{2} q^{2}}{8}\right] d F(q)-k=\frac{\theta^{2}}{24}-k$, and the retailer's equilibrium interim payoff is $\bar{\pi}=\int_{0}^{1}\left[\frac{\theta^{2} q^{2}}{16}\right] d F(q)=\frac{\theta^{2}}{48}$. Thus, it is evident that with mandatory acquisition, the cost of quality information acquisition $k$ should be lower than $\frac{\theta^{2}}{24}$. Otherwise, the manufacturer cannot afford the information acquisition cost and may quit the market. This case may represent practices in some industries for which, due to safety concerns, regulators require a full disclosure of product quality information for companies that want to enter the market. This may result in high quality acquisition costs that prevent small companies from entering the market. Given the quality information acquisition cost distribution, we can derive the manufacturer's ex-ante payoff as $E[\bar{\Pi}]=\int_{0}^{\frac{\theta^{2}}{24}}\left(\frac{\theta^{2}}{24}-k\right) d G(k)=\frac{1}{2 K}\left(\frac{\theta^{2}}{24}\right)^{2}$, and the retailer's ex-ante payoff can be expressed as $E[\bar{\pi}]=\int_{0}^{\frac{\theta^{2}}{24}}\left(\frac{\theta^{2}}{48}\right) d G(k)=\frac{1}{2 K}\left(\frac{\theta^{2}}{24}\right)^{2}$. Similarly, the expected consumer surplus with mandatory acquisition of product quality information is given by $\overline{E C S}=\int_{0}^{\frac{\theta^{2}}{24}} \int_{0}^{1} \frac{\theta^{2} q^{2}}{32} d F(q) d G(k)=\frac{1}{4 K}\left(\frac{\theta^{2}}{24}\right)^{2}$. We then compare the firms' ex-ante payoffs and expected consumer surplus with those of the basic model (shown in Appendix B in the Supplement File). The results are shown in Proposition 9 and illustrated in the following figure.

Proposition 9. With mandatory acquisition of product quality information, the manufacturer is ex-ante worse off, while the retailer's ex-ante payoff and the expected consumer surplus may become better off.
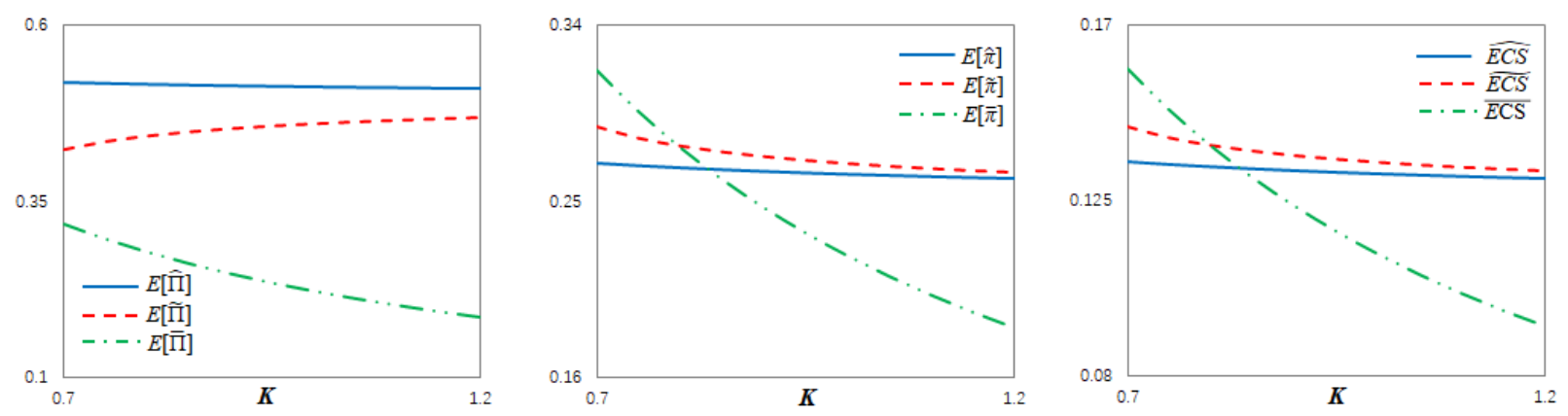

Figure 9 The comparison of the manufacturer's ex-ante payoff, the retailer's ex-ante payoff and the expected consumer surplus under mandatory acquisition and those under the two benchmark cases (here, $\theta=4)$

It is evident that the manufacturer is worse off under mandatory acquisition of product quality information, as this eliminates flexibility to engage in quality information acquisition. Moreover, when the cost of quality information acquisition is sufficiently high, the manufacturer chooses to quit the market and obtains zero payoff. Mandatory acquisition can be a double-edged sword for either the retailer or the consumers. On the one hand, it ensures perfect quality information transparency in the supply chain when the information acquisition cost is relatively low, which is beneficial to both the retailer and the consumers. On the other hand, it may also exclude the 
manufacturer when the information acquisition cost is high, hurting both the retailer and the consumers. As shown in Figure 9, the retailer and the consumers can benefit from mandatory acquisition only if the upper bound of quality information acquisition cost $(K)$ is relatively low. Under such a circumstance, the manufacturer is more likely to undertake quality information acquisition than to leave the market. That is there are cases (i.e., the upper bound of quality information acquisition cost $K$ is low) where government intervention (i.e., both the acquisition and disclosure of product quality information are mandatory) is necessary to protect the consumers for some critical products like those requiring RoSH certification, at the costs from the manufacturer.

\section{Concluding Remarks}

This study investigates the impacts of mandatory and voluntary disclosure of product quality information on an upstream manufacturer's quality information acquisition strategies in a decentralized supply chain. Product quality is initially uncertain for the manufacturer, the retailer and the consumers, while the manufacturer can privately acquire the precise quality information of his product at a certain cost that is unobservable to the retailer and the consumers. After quality information acquisition, the manufacturer decides whether to disclose the quality information to the unknown retailer and consumers, depending on the quality information disclosure systems to which the quality information acquisition process is subject. We show that different quality information disclosure systems significantly change the manufacturer's equilibrium quality information acquisition strategies. In particular, voluntary disclosure allows for the manufacturer to craft his disclosure strategy based on the realized quality information, which in turn, gives the manufacturer a stronger incentive to acquire the precise quality information of his product. In contrast, mandatory disclosure eliminates the manufacturer's chance of strategic quality information withholding and thus makes the manufacturer more conservative in acquiring such quality information.

The change in equilibrium quality information acquisition strategies also gives rise to some unintended consequences for the profitability of the manufacturer, retailer and consumer. First, we show that mandatory disclosure is more beneficial to the manufacturer than voluntary disclosure. This can be explained from the perspective that although mandatory disclosure makes the manufacturer inflexible at crafting the quality information disclosure strategy, it also hinders the consumer's quality speculation process. This reversely pulls up the consumer's quality expectations upon non-disclosure and improves the manufacturer's ex-ante payoff. Second, both the retailer and the consumers prefer voluntary disclosure whenever voluntary disclosure induces 
the manufacturer to acquire the precise quality information about his product. This outcome is because voluntary disclosure makes the manufacturer more active in acquiring and sharing quality information, leading to a higher extent of quality information transparency in the supply chain. Thus, both the retailer and the consumers benefit from making more precise pricing and purchasing decisions in such an environment.

We also conduct several extensions to verify the robustness of our main results. In particular, we assume that the manufacturer may privately know product quality information with some probability, and we show that he still has a stronger quality information acquisition incentive but obtains a lower ex-ante payoff under voluntary disclosure than under mandatory disclosure. We also consider some variations about the demand function form and the distributions of product quality and quality information acquisition cost, wherein the main results in the basic model still hold with these modifications. Note that the ratio between the cost of quality information acquisition and the manufacturer's payoff is examined too, in which we show that even for a relatively small quality information acquisition cost, it can still influence the manufacturer's equilibrium quality information acquisition strategy and payoff.

It is worth mentioning that in our paper, the consumer is restricted to obtain precise product quality information only via the manufacturer's quality information acquisition and disclosure behavior. While with the development of information technology, nowadays the consumer may acquire product quality information via some other methods, such as consumer review. We believe that combining the consumer review and the manufacturer's voluntary quality information acquisition/disclosure behavior should be a very interesting direction, but it is also very challenging and may require a fundamental modification to the current work. For example, to explore the impact of consumer review on the manufacturer's equilibrium quality information acquisition/disclosure strategies, we may need to extend the current one-period model into a two-period game to ensure that the first-arriving consumers can generate their reviews in the first period and then the late-arriving consumers can update their quality expectation after observing these reviews. During this process, how different consumers generate their reviews is also questionable, given that a review contains not only the quality information but also the consumer's private preference information. In this sense, it is not clear whether the strategic impact of a quality information disclosure system on the firm's quality information acquisition/disclosure strategy and payoff still remains. Therefore, we think that it may deserve a separate study. There are also some other possible directions for future research. One good candidate is to examine the influence of upstream competition on the manufacturers' incentives to acquire product quality information in a supply chain with different quality information disclosure systems. Another direction is to 
consider heterogeneous consumers. It would be interesting to study the interactions between the manufacturer's information acquisition strategies and different information disclosure systems for product quality in a market where a fraction of consumers cares about the product quality, while the rest do not. We plan to investigate these issues in our future research.

\section{Appendix A}

\section{Proof of the Existence and Uniqueness of $\alpha$ in Proposition 1}

To simplify analysis, let $t=\sqrt{1-\alpha}$. Note that $t$ strictly decreases in $\alpha$, with $\alpha \in(0,1), t \in(0,1)$. We could write $q_{n d}$ and $\tilde{k}$ as: $q_{n d}=\frac{\sqrt{1-\alpha}-(1-\alpha)}{\alpha}=\frac{t}{1+t}$ and $\tilde{k}=\frac{\theta^{2}\left[2\left(q_{n d}\right)^{3}-3\left(q_{n d}\right)^{2}+1\right]}{24}=\frac{\theta^{2}(3 t+1)}{24(1+t)^{3}}$. Since $\alpha=\operatorname{Pr}(k \leq \widetilde{k})$, we can obtain the equation: $\frac{\left(1-t^{2}\right)(1+t)^{3}}{3 t+1}=\frac{\theta^{2}}{24 K}$. Define the left-hand side of this equation as $\psi(t)$, which is a continuous function for $t \in(0,1)$. As $\psi^{\prime}(t)=\frac{4 t(1-3 t)(1+t)^{3}}{(3 t+1)^{2}}, \psi(t)$ is strictly increasing for $t \in\left(0, \frac{1}{3}\right]$ and strictly decreasing for $t \in\left[\frac{1}{3}, 1\right)$. Note that when $t \rightarrow 0$, $\psi(t) \rightarrow 1$, and when $t \rightarrow 1, \psi(t) \rightarrow 0$. Note also that the right-hand side of the above equation takes a value in the range $(0,1)$ with $K>\frac{\theta^{2}}{24}$. Thus, there exists a unique solution to $t$ that is in the range $(0,1)$. Consequently, there is a unique solution to $\alpha$.

\section{Proof of Proposition 4}

As has been discussed in the text, the manufacturer's ex-ante payoff under voluntary disclosure is $E[\widetilde{\Pi}]=G(\widetilde{k})\left[\int_{0}^{q_{n d}}\left(\widetilde{\Pi}_{n d}\right) d F(q)+\int_{q_{n d}}^{1}\left(\widetilde{\Pi}_{d}\right) d F(q)\right]+[1-G(\widetilde{k})] \widetilde{\Pi}_{n d}-\int_{0}^{\widetilde{k}} k d G(k)=\frac{\widetilde{k}^{2}}{2 K}+\frac{\theta^{2}\left(q_{n d}\right)^{2}}{8}$, and the manufacturer's ex-ante payoff under mandatory disclosure is $E[\widehat{\Pi}]=G(\widehat{k}) \int_{0}^{1}\left(\widehat{\Pi}_{d}\right) d F(q)+$ $[1-G(\widehat{k})] \widehat{\Pi}_{n d}-\int_{0}^{\widehat{k}} k d G(k)=\frac{\widehat{k}^{2}}{2 K}+\frac{\theta^{2} \bar{q}^{2}}{8}$, where $\bar{q}=\frac{1}{2}$. The difference of the two ex-ante payoffs is $\Delta \Pi=E[\widehat{\Pi}]-E[\widetilde{\Pi}]=\frac{\widehat{k}^{2}-\widetilde{k}^{2}}{2 K}+\frac{\theta^{2}\left[\bar{q}^{2}-\left(q_{n d}\right)^{2}\right]}{8}$. Applying the expressions of $q_{n d}$ and $\widetilde{k}$ with respect to $t$ (from the preceding proof), the difference of the two payoffs can be expressed as $\Delta \Pi=\frac{1}{2}\left[\frac{\theta^{2}}{24 K}\right.$. $\left.\frac{\theta^{2}}{384}-\frac{\theta^{2}}{24 K} \cdot \frac{(3 t+1)}{(1+t)^{3}} \cdot \frac{\theta^{2}}{24} \cdot \frac{(3 t+1)}{(1+t)^{3}}\right]+\frac{\theta^{2}}{32}\left[1-\frac{4 t^{2}}{(1+t)^{2}}\right]$. Also from the preceding proof, we have $\frac{\left(1-t^{2}\right)(1+t)^{3}}{3 t+1}=$ $\frac{\theta^{2}}{24 K}$. Thus,

$$
\begin{aligned}
\Delta \Pi & =\frac{1}{2}\left[\frac{\left(1-t^{2}\right)(1+t)^{3}}{3 t+1} \cdot \frac{\theta^{2}}{384}-\frac{\left(1-t^{2}\right)(3 t+1)}{(1+t)^{3}} \cdot \frac{\theta^{2}}{24}\right]+\frac{\theta^{2}}{32} \cdot \frac{\left(1-t^{2}\right)(3 t+1)}{(1+t)^{3}} \\
& =\frac{\theta^{2}\left(1-t^{2}\right)}{768}\left[\frac{(1+t)^{3}}{3 t+1}+\frac{8(3 t+1)}{(1+t)^{3}}\right]>0,
\end{aligned}
$$

and we can demonstrate that the manufacturer's ex-ante payoff is higher under mandatory disclosure.

For the retailer, her ex-ante payoff, with voluntary disclosure of quality information, is $E[\tilde{\pi}]=$ $G(\widetilde{k})\left[\int_{0}^{q_{n d}}\left(\widetilde{\pi}_{n d}\right) d F(q)+\int_{q_{n d}}^{1}\left(\widetilde{\pi}_{d}\right) d F(q)\right]+[1-G(\widetilde{k})] \widetilde{\pi}_{n d}=\frac{\widetilde{k}^{2}}{2 K}+\frac{\theta^{2}\left(q_{n d}\right)^{2}}{16}$, and her ex-ante payoff under mandatory disclosure is $E[\widehat{\pi}]=G(\widehat{k}) \int_{0}^{1}\left(\widehat{\pi}_{d}\right) d F(q)+[1-G(\widehat{k})] \widehat{\pi}_{n d}=\frac{\widehat{k}^{2}}{2 K}+\frac{\theta^{2} \bar{q}^{2}}{16}$. We will first 
compare the total ex-ante payoffs of the firms in supply chain, that is, the sum of ex-ante payoffs earned by the manufacturer and the retailer, under voluntary disclosure and mandatory disclosure. Using the same method as that in the comparison of the manufacturer's ex-ante payoffs, we have $\Delta(\Pi+\pi)=(E[\widehat{\Pi}]+E[\widehat{\pi}])-(E[\widetilde{\Pi}]+E[\widetilde{\pi}])=\frac{\widehat{k}^{2}-\widetilde{k}^{2}}{K}+\frac{3 \theta^{2}\left[\bar{q}^{2}-\left(q_{n d}\right)^{2}\right]}{16}$. Substituting the expressions of $q_{n d}$ and $\widetilde{k}$ with respect to $t$ into $\Delta(\Pi+\pi)$, we obtain that

$$
\begin{aligned}
\Delta(\Pi+\pi) & =\left[\frac{\left(1-t^{2}\right)(1+t)^{3}}{3 t+1} \cdot \frac{\theta^{2}}{384}-\frac{\left(1-t^{2}\right)(3 t+1)}{(1+t)^{3}} \cdot \frac{\theta^{2}}{24}\right]+\frac{3 \theta^{2}}{64} \cdot \frac{\left(1-t^{2}\right)(3 t+1)}{(1+t)^{3}} \\
& =\frac{\theta^{2}\left(1-t^{2}\right)}{384}\left[\frac{(1+t)^{3}}{3 t+1}+\frac{2(3 t+1)}{(1+t)^{3}}\right]>0 .
\end{aligned}
$$

Back to the comparison of the retailer's ex-ante payoffs, we have $\Delta \pi=\Delta(\Pi+\pi)-\Delta \Pi=$ $\frac{\theta^{2}\left(1-t^{2}\right)}{768}\left[\frac{(1+t)^{3}}{3 t+1}-\frac{4(3 t+1)}{(1+t)^{3}}\right]$. The inequality is implied by the fact that the term in the brackets is negative since $(1+t)^{6}<4(3 t+1)^{2}$ for $t \in(0,1)$. Therefore, $\Delta \pi<0$, the retailer's ex-ante payoff is higher under voluntary disclosure. The proof of Proposition 4 is completed.

\section{Proof of Proposition 5}

As has been discussed in our paper, the expected consumer surplus when the disclosure of product quality information is voluntary is $\widetilde{E C S}=G(\widetilde{k})\left[\int_{0}^{q_{n d}}\left(\widetilde{C S}_{n d}\right) d F(q)+\int_{q_{n d}}^{1}\left(\widetilde{C S}_{d}\right) d F(q)\right]+[1-$ $G(\widetilde{k})] \widetilde{C S}_{n d}=\frac{\widetilde{k}^{2}}{4 K}+\frac{\theta^{2}\left(q_{n d}\right)^{2}}{32}$, and with mandatory disclosure of quality information, the expected consumer surplus is $\widehat{E C S}=G(\widehat{k}) \int_{0}^{1}\left(\widehat{C S}_{d}\right) d F(q)+[1-G(\widehat{k})] \widehat{C S}_{n d}=\frac{\widehat{k}^{2}}{4 K}+\frac{\theta^{2} \bar{q}^{2}}{32}$. The difference of the expected consumer surplus is $\triangle E C S=\widehat{E C S}-\widetilde{E C S}=\frac{\widehat{k}^{2}-\widetilde{k}^{2}}{4 K}+\frac{\theta^{2}\left[\bar{q}^{2}-\left(q_{n d}\right)^{2}\right]}{32}$. Applying the expressions of $q_{n d}$ and $\widetilde{k}$ with respect to $t$ (from the preceding proofs of Proposition 4 ), we obtain that

$$
\begin{aligned}
\Delta E C S & =\frac{1}{4}\left[\frac{\left(1-t^{2}\right)(1+t)^{3}}{3 t+1} \cdot \frac{\theta^{2}}{384}-\frac{\left(1-t^{2}\right)(3 t+1)}{(1+t)^{3}} \cdot \frac{\theta^{2}}{24}+\frac{(1-t)(3 t+1)}{(1+t)^{2}} \cdot \frac{\theta^{2}}{32}\right] \\
& =\frac{\theta^{2}\left(1-t^{2}\right)}{1536} \cdot\left[\frac{(1+t)^{3}}{3 t+1}-\frac{4(3 t+1)}{(1+t)^{3}}\right] .
\end{aligned}
$$

As $(1+t)^{6}<4(3 t+1)^{2}$ for $t \in(0,1)$, thus $\frac{(1+t)^{3}}{3 t+1}-\frac{4(3 t+1)}{(1+t)^{3}}<0$, and $\widehat{E C S}-\widetilde{E C S}<0$. Therefore, we can demonstrate that the expected consumer surplus under voluntary disclosure is higher than that under mandatory disclosure, completing the proof of Proposition 5.

\section{Proofs of Proposition 6 and Proposition 7}

Under voluntary disclosure, in the first stage, the ex-ante uninformed manufacturer's payoff from acquisition and no acquisition are given by $\int_{0}^{\tilde{q}_{n d}}\left[\frac{\theta^{2}\left(\widetilde{q}_{n d}\right)^{2}}{8}\right] d F(q)+\int_{\widetilde{q}_{n d}}^{1}\left(\frac{\theta^{2} q^{2}}{8}\right) d F(q)-k$ and $\frac{\theta^{2}\left(\widetilde{q}_{n d}\right)^{2}}{8}$. The manufacturer chooses to acquire product quality information only if it is profitable, leading 
to the essential condition that $k \leq \widetilde{k}=\frac{\theta^{2}\left[2\left(\widetilde{q}_{n d}\right)^{3}-3\left(\widetilde{q}_{n d}\right)^{2}+1\right]}{24}$. Let $t=\sqrt{1-[(1-\rho) \alpha+\rho]}$, then we could rewrite $\widetilde{q}_{n d}$ and $\widetilde{k}$ as $\widetilde{q}_{n d}=\frac{t}{1+t}$ and $\widetilde{k}=\frac{\theta^{2}}{24}\left[2\left(\frac{t}{1+t}\right)^{3}-3\left(\frac{t}{1+t}\right)^{2}+1\right]=\frac{\theta^{2}(3 t+1)}{24(1+t)^{3}}$, where $t \in(0, \sqrt{1-\rho})$. As $\alpha=\frac{(1-\rho)-t^{2}}{1-\rho}=1-\frac{t^{2}}{1-\rho}$, we obtain the equation: $\frac{\left[(1-\rho)-t^{2}\right](1+t)^{3}}{(1-\rho)(3 t+1)}=\frac{\theta^{2}}{24 K}$, where $t \in(0, \sqrt{1-\rho})$. Define $\varphi(t)=\frac{\left[(1-\rho)-t^{2}\right](1+t)^{3}}{(1-\rho)(3 t+1)}$, which is a continuous function for $t \in(0, \sqrt{1-\rho})$, and $\varphi^{\prime}(t)=\frac{2 t(1+t)^{2}\left[3(1-\rho)-1-4 t-6 t^{2}\right]}{(1-\rho)(3 t+1)^{2}}$. Let $\phi(t)=3(1-\rho)-1-4 t-6 t^{2}$. As $\phi^{\prime}(t)=-4-12 t<0$ for $t \in(0, \sqrt{1-\rho}), \phi(t)$ is strictly decreasing in $t$ for $t \in(0, \sqrt{1-\rho})$. When $t \rightarrow \sqrt{1-\rho}, \phi(t)=$ $-3(1-\rho)-4 \sqrt{1-\rho}-1<0$; when $t \rightarrow 0, \phi(t)=3(1-\rho)-1$ : (i) if $0<\rho<\frac{2}{3}, \phi(t)>0$ when $t \rightarrow 0$, then there exists a unique $t^{*}$ satisfying $\phi\left(t^{*}\right)=0$ given that $\phi(t)<0$ when $t \rightarrow \sqrt{1-\rho}$, thus $\varphi^{\prime}(t) \geq 0$ for $t \in\left(0, t^{*}\right]$ and $\varphi^{\prime}(t) \leq 0$ for $t \in\left[t^{*}, \sqrt{1-\rho}\right), \varphi(t)$ is increasing for $t \in\left(0, t^{*}\right]$ and decreasing for $t \in\left[t^{*}, \sqrt{1-\rho}\right)$, note that the left hand side of the equation $\varphi(t) \rightarrow 1$ when $t \rightarrow 0$, and $\varphi(t) \rightarrow 0$ when $t \rightarrow \sqrt{1-\rho}$, with $K>\frac{\theta^{2}}{24}$, the right-hand side of the equation takes a value in the range $(0,1)$, thus there exists a unique solution to $t$ on the interval $(0, \sqrt{1-\rho})$; (ii) if $\frac{2}{3} \leq \rho<1$, $\phi(t) \leq 0$ for $t \in(0, \sqrt{1-\rho})$, thus $\varphi^{\prime}(t) \leq 0$ and $\varphi(t)$ is decreasing in $t$ for $t \in(0, \sqrt{1-\rho})$, then note that when $t \rightarrow 0$, the left hand side of the equation $\varphi(t) \rightarrow 1$, and when $t \rightarrow \sqrt{1-\rho}$, $\varphi(t) \rightarrow 0$, note also that the right-hand side of the equation takes a value in the range $(0,1)$ with $K>\frac{\theta^{2}}{24}$. Thus, there exists a unique solution to $t$ that is in the range $(0, \sqrt{1-\rho})$. Consequently, both $q_{n d}$ and $\tilde{k}$ are well defined, completing the proof of Proposition 6.

Under mandatory disclosure, in the first stage, the ex-ante uninformed manufacturer's payoff from acquisition and no acquisition are given by $\int_{0}^{1}\left(\frac{\theta^{2} q^{2}}{8}\right) d F(q)-k$ and $\frac{\theta^{2}\left(\widehat{q}_{n d}\right)^{2}}{8}$ respectively. The manufacturer chooses to acquire product quality information only if it is profitable, leading to the essential condition that $k \leq \widehat{k}=\frac{\theta^{2}\left[1-3\left(\widehat{q}_{n d}\right)^{2}\right]}{24}$, where $\widehat{q}_{n d}=\frac{\sqrt{1-\rho}-(1-\rho)}{\rho}$, completing the proof of Proposition 7.

\section{References}

Arya, A., N. Gong, R. N. Ramanan. 2014. Quality testing and product rationing by input suppliers. Production and Operations Management, 23(11), 1835-1844.

Board, O. 2009. Competition and disclosure. Journal of Industrial Economics, 57(1), 197-213.

Cheong, I., J. Y. Kim. 2004. Costly information disclosure in oligopoly. Journal of Industrial Economics, 52(1), 121-132.

Dahm, M., P. Gonzlez, N. Porteiro. 2009. Trials, tricks and transparency: How disclosure rules affect clinical knowledge. Journal of Health Economics, 28(6), 1141-1153.

Dahm, M., P. Gonzlez, N. Porteiro. 2016. The enforcement of mandatory disclosure rules. Journal of Public Economics, 167, 21-32. 
Dafny, L., D. Dranove. 2008. Do report cards tell consumers anything they don't already know? The case of Medicare HMOs. RAND Journal of Economics, 39(3), 790-821.

Fishman, M. J., K. M. Hagerty. 2003. Mandatory versus voluntary disclosure in markets with informed and uninformed customers. Journal of Law, Economics, and organization, 19(1), 45-63.

Gao, L., Z. Li, B. Shou. 2014. Information acquisition and voluntary disclosure in an exportprocessing system. Production and Operations Management, 23(5), 802-816.

Ghosh, B., M. R. Galbreth. 2013. The impact of consumer attentiveness and search costs on firm quality disclosure: A competitive analysis. Management Science, 59(11), 2604-2621.

Grossman, S. J. 1981. The informational role of warranties and private disclosure about product quality. Journal of Law and Economics, 24(3), 461-483.

Grossman, S. J., O. D. Hart. 1980. Disclosure laws and takeover bids. Journal of Finance, 35(2), 323-334.

Guan, X., Y. J. Chen. 2015. Hierarchical quality disclosure in a supply chain with cost heterogeneity. Decision Support Systems, 76, 63-75.

Guan, X., Y. J. Chen. 2017. The interplay between information acquisition and quality disclosure. Production and Operations Management, 26(3), 389-408.

Guo, L. 2009. The benefits of downstream information acquisition. Marketing Science, 28(3), 457471.

Guo, L., G. Iyer. 2010. Information acquisition and sharing in a vertical relationship. Marketing Science, 29(3), 483-506.

Guo, L., Y. Zhao. 2009. Voluntary quality disclosure and market interaction. Marketing Science, 28(3), 488-501.

Ha, A. Y., S. Tong, H. Zhang. 2011. Sharing demand information in competing supply chains with production diseconomies. Management Science, 57(3), 566-581.

Iyer, G., S. Singh. 2018. Voluntary product safety certification. Management Science, 64(2), 695-714.

Jansen, J. 2008. Information acquisition and strategic disclosure in oligopoly. Journal of Economics \& Management Strategy, 17(1), 113-148.

Jiang, B., L. Tian, Y. Xu, F. Zhang. 2016. To share or not to share: Demand forecast sharing in a distribution channel. Marketing Science, 35(5), 800-809.

Jin, G. Z., Leslie, P. 2003. The effect of information on product quality: Evidence from restaurant 
hygiene grade cards. The Quarterly Journal of Economics, 118(2), 409-451.

Jovanovic, B. 1982. Truthful disclosure of information. Bell Journal of Economics, 13(1), 36-44.

Levin, D., J. Peck, L. Ye. 2009. Quality disclosure and competition. Journal of Industrial Economics, 57(1), 167-196.

Li, T., S. Tong, H. Zhang. 2014. Transparency of information acquisition in a supply chain. Manufacturing \& Service Operations Management, 16(3), 412-424.

Li, X., R. Peeters. 2017. Rivalry information acquisition and disclosure. Journal of Economics \& Management Strategy, 26(3), 610-623.

Mishra, B. K., S. Raghunathan, X. Yue. 2009. Demand forecast sharing in supply chains. Production and Operations Management, 18(2), 152-166.

Matthews, S., A. Postlewaite. 1985. Quality testing and disclosure. RAND Journal of Economics, 16(3), 328-340.

Markopoulos, P. M., K. Hosanagar. 2017. A model of product design and information disclosure investments. Management Science, 64(2), 739-759.

Milgrom, P. R. 1981. Good news and bad news: Representation theorems and applications. Bell Journal of Economics, 12(2), 380-391.

Shang, W., A. Y. Ha, S. Tong. 2015. Information sharing in a supply chain with a common retailer. Management Science, 62(1), 245-263.

Shavell, S. 1994. Acquisition and disclosure of information prior to sale. RAND Journal of Economics, 25(1), 20-36.

Singh, N., X. Vives. 1984. Price and quantity competition in a differentiated duopoly. RAND Journal of Economics, 15(4), 546-554.

Sun, M. 2011. Disclosing multiple product attributes. Journal of Economics \& Management Strategy, 20(1), 195-224.

Yehezkel, Y. 2014. Motivating a supplier to test product quality. The Journal of Industrial Economics, 62(2), 309-345.

Zhang, H. 2002. Vertical information exchange in a supply chain with duopoly retailers. Production and Operations Management, 11(4), 531-546. 